\title{
Shape sensitivity of eigenvalues in hydrodynamic stability, with physical interpretation for the flow around a cylinder
}

\author{
Jack Brewster ${ }^{1}$, Matthew P. Juniper ${ }^{1, *}$ \\ Cambridge University Engineering Department, Trumpington Street, Cambridge CB2 1PZ, United Kingdom
}

\begin{abstract}
The shape gradients of an instability's growth rate and frequency are derived for an unstable mode calculated from a global stability analysis. These are calculated and interpreted physically for the flow around a cylinder at a Reynolds number of 50. This is a well-known canonical flow, which is often used to discover fundamental behaviour in bluff body flows and to test new numerical techniques. This paper shows that shape deformations affect hydrodynamic oscillations mainly through their influence on the steady base-flow, rather than through their influence on the unsteady feedback mechanism. Deformations that strongly affect the base-flow are shown to strongly affect the frequency and growth rate, as expected. In addition, subtle deformations at the rear of the cylinder are shown to exploit small base-flow changes that have a disproportionately large influence on the growth rate. The physical mechanism behind this is shown to be similar to the well-known phenomenon of 'base bleed'. The method presented in this paper is general and versatile. It provides engineers with gradient information in order to optimize designs systematically. In addition, it provides physical insight, which enables intuitive design changes that would be outside the range of an optimization algorithm or existing geometric parametrization.
\end{abstract}

Keywords: Vortex shedding

Absolute/convective instability

Instability control

\section{Introduction}

Many flows are unstable and some oscillate robustly at well-defined frequencies. These oscillations are sometimes desirable, for example in vortex shedding flowmeters [1], and sometimes undesirable, for example in cyclonic separators [2]. In many cases, a designer would like to alter the growth rate or frequency of these hydrodynamic oscillations by changing the shape of an object. Previously, shape changes to stabilise the flow or to change the oscillation frequency were tried one by one, either numerically [3] or experimentally. This is too expensive for practical applications where geometries may be described by many hundreds of parameters. Adjoint-based shape optimization techniques that efficiently calculate the shape gradient of an instability's growth rate have recently been introduced in the literature $[4,5]$. These studies focus solely on the growth-rate of the instability and treat the shape gradient as a black box in an optimization algorithm. The purpose of this paper is to extend these techniques, deriving both the shape gradient of the instability's growth rate and frequency, and to provide physical interpretation. This physical insight enables engineers to perform intuitive design changes that would be outside the range of an

\footnotetext{
* Corresponding author

Email address: mpj1001@cam.ac.uk (Matthew P. Juniper)
}

optimization algorithm or existing geometric parametrization. These techniques are demonstrated successfully on the canonical case of flow around a cylinder at a Reynolds number of 50, opening up the possibility for its application to other flows.

Above a critical Reynolds number $R e_{c} \approx 47$, the steady flow around a 2D cylinder becomes unstable to hydrodynamic oscillations at a well-defined Strouhal number [6]. These oscillations are caused by a sufficiently large region of local absolute instability in the wake of the cylinder $[7,8,9]$. A linear stability analysis of small perturbations to the $2 \mathrm{D}$ steady flow leads to an eigenvalue problem for the perturbation's frequency and growth rate (the eigenvalue) and spatial structure (the eigenfunction) [10]. The spatial structure, which is often called the direct global mode, is given by the right eigenfunction. The receptivity of the flow to external forcing, which is often called the adjoint global mode, is given by the left eigenfunction. Overlapping the direct and adjoint global modes gives the structural sensitivity, which quantifies the eigenvalue's sensitivity to localized unsteady feedback $[10,11]$. Combining these with the adjoint of the base-flow [6] gives the base-flow sensitivity, which quantifies the eigenvalue's sensitivity to localized changes to the base-flow.

The structural sensitivity and the base-flow sensitivity have been used to investigate open loop control schemes 
for the flow oscillations around a cylinder [12]. Examples include modification of the base-flow [12], boundary blowing and suction [13], and placement of a small cylinder just downstream $[6,10,11,14]$. In many practical situations, however, one would like to alter the eigenvalue by changing the shape of the object itself, rather than by blowing or sucking through its surface. Examples where this would be useful are control of the precessing vortex core in cyclonic separators [2] and control of hydrodynamic oscillations in gas turbine fuel injectors $[15,16,17]$. The sensitivity of the eigenvalue to shape changes could be calculated by finite differences [3] but this is too expensive to be incorporated into a gradient-based optimization algorithm. The eigenvalue sensitivity can also be calculated using adjoint methods by combining the framework of the structural and base-flow sensitivity with the techniques pioneered by Pironneau [18] for the shape optimization of hydrodynamic systems. This was done using a Lagrange multiplier method for the flow in a channel containing a rapid expansion by Nakazawa \& Azegami [4] and recently applied for the case of a cylinder by Kiriyama et. al. [5]. These papers contain no physical interpretation, however, and consider only the growth rate sensitivity.

This paper calculates the sensitivity to shape changes of growth rates and frequencies for the flow around a cylinder at $\operatorname{Re}=50$. This is significantly more challenging than calculating the sensitivity to base-flow modifications, which has been done by Marquet et al. [6], because the computational domain changes. The paper shows that two types of shape deformation contribute to the eigenvalue sensitivities: (i) deformations that cause a large change to the baseflow, which then inefficiently alter the eigenvalue, and (ii) deformations that cause a small change to the base-flow, which then efficiently alter the eigenvalue. These terms are interpreted physically and then combined with information about how the deformations alter the production and advection of perturbations. This allows the physical mechanisms causing the eigenvalue sensitivity to be interpreted in terms of existing observations, such as the fact that instability can be reduced by bleeding flow slowly into the recirculating zone [19], which is known as 'base bleed'.

\section{Mathematical framework}

We consider the viscous open flow past an object (figure 1). The state variable, $\mathbf{q} \equiv[\mathbf{u}, p]^{T}$, contains the velocity field, $\mathbf{u}$, and the pressure field, $p$. The state is governed by the incompressible Navier-Stokes equations. The far-field boundary conditions are: a prescribed velocity, $\mathbf{u}_{+}$, on the inlet, $\Gamma_{+}$; a no-stress condition on the outlet, $\Gamma_{-}$; and perfect-slip conditions on the lateral boundaries, $\Gamma_{s}$. Inside the domain, a closed no-slip boundary, $\Gamma_{0}$, defines the shape of the object. The governing equations and boundary conditions are written:

$$
\mathcal{N}(\mathbf{q})=\left(\begin{array}{c}
\frac{\partial \mathbf{u}}{\partial t}+\nabla \mathbf{u} \cdot \mathbf{u}+\nabla p-\nu \nabla^{2} \mathbf{u} \\
\nabla \cdot \mathbf{u}
\end{array}\right)=0
$$

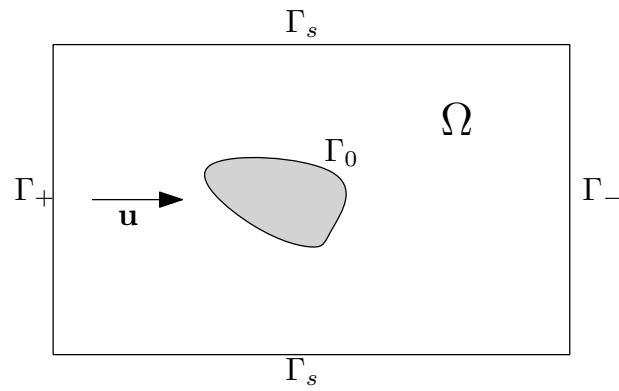

Figure 1: The generic domain, $\Omega$, represents the viscous open flow over an object. The external boundary of the domain consists of an inlet $\left(\Gamma_{+}\right)$, outlet $\left(\Gamma_{-}\right)$and perfect-slip $\left(\Gamma_{s}\right)$ surface. A no-slip surface $\left(\Gamma_{0}\right)$ defines the shape of the object.

$$
\begin{gathered}
\mathbf{u}=\mathbf{u}_{+} \text {on } \Gamma_{+} ; \quad p \tilde{\mathbf{n}}-\nu \frac{\partial \mathbf{u}}{\partial n}=0 \text { on } \Gamma_{-} ; \\
\frac{\partial \mathbf{u}}{\partial n} \cdot \tilde{\boldsymbol{\tau}}=\mathbf{u} \cdot \tilde{\mathbf{n}}=0 \text { on } \Gamma_{s} ; \quad \mathbf{u}=0 \text { on } \Gamma_{0} .
\end{gathered}
$$

We use the tensorial notation, $(\nabla \mathbf{u})_{i j} \equiv \frac{\partial u_{i}}{\partial x_{j}}$. The unit normal and unit tangent vectors are $\tilde{\mathbf{n}}$ and $\tilde{\boldsymbol{\tau}}$. The spatial derivative of $\mathbf{u}$ in the normal direction is $\frac{\partial \mathbf{u}}{\partial n} \equiv \nabla \mathbf{u} \cdot \tilde{\mathbf{n}}$ and in the tangential direction is $\frac{\partial \mathbf{u}}{\partial \tau} \equiv \nabla \mathbf{u} \cdot \tilde{\boldsymbol{\tau}}$.

The base-flow, $\overline{\mathbf{q}}$, is a steady solution to (1). The baseflow satisfies the no-slip condition on $\Gamma_{0}$ and so is a function of $\Gamma_{0}$, i.e. $\overline{\mathbf{q}} \equiv \overline{\mathbf{q}}\left(\Gamma_{0}\right)$.

We perform a global linear stability analysis by adding to the base-flow an infinitesimal perturbation of the form $\hat{\mathbf{q}} \mathrm{e}^{s t}$, where $\hat{\mathbf{q}}$ and $s$ are both complex. The governing equations for $\hat{\mathbf{q}}$ and $s$ are the Navier-Stokes equations linearized about $\overline{\mathbf{q}}$ :

$$
\begin{gathered}
s\left(\begin{array}{c}
\hat{\mathbf{u}} \\
0
\end{array}\right)+\mathcal{L} \hat{\mathbf{q}}=0 \quad ; \\
\text { where } \mathcal{L} \hat{\mathbf{q}} \equiv\left(\begin{array}{cc}
\nabla \star \cdot \overline{\mathbf{u}}+\nabla \overline{\mathbf{u}} \cdot \star-\nu \nabla^{2} \star & \nabla \star \\
\nabla \cdot \star & 0
\end{array}\right)\left(\begin{array}{c}
\hat{\mathbf{u}} \\
\hat{p}
\end{array}\right) ; \\
\hat{\mathbf{u}}=0 \text { on } \Gamma_{+} ; \quad \hat{p} \tilde{\mathbf{n}}-\nu \frac{\partial \hat{\mathbf{u}}}{\partial n}=0 \text { on } \Gamma_{-} ; \\
\frac{\partial \hat{\mathbf{u}}}{\partial n} \cdot \tilde{\boldsymbol{\tau}}=\hat{\mathbf{u}} \cdot \tilde{\mathbf{n}}=0 \text { on } \Gamma_{s} ; \quad \hat{\mathbf{u}}=0 \text { on } \Gamma_{0} .
\end{gathered}
$$

This is an eigenvalue problem whose solution is a series of eigenmodes, which are ordered pairs, $(\hat{\mathbf{q}}, s)$, consisting of a complex eigenfunction, $\hat{\mathbf{q}}$, and its corresponding complex eigenvalue, $s$. The real part of $s$ is the eigenmode's growth rate, $\sigma$, and the imaginary part is the eigenmode's frequency, $\omega$. The sensitivity analysis that follows is specific to a chosen eigenmode, typically that with the largest growth rate. We normalise the eigenfunction by setting $\|\hat{\mathbf{u}}\|_{\Omega}=1$, where $\|\star\|_{\Omega}$ is the norm corresponding to the inner product, $\langle\mathbf{a}, \mathbf{b}\rangle_{\Omega} \equiv \int_{\Omega} \mathbf{a}^{*} \cdot \mathbf{b} \mathrm{d} \Omega$.

The eigenfunction satisfies a no-slip condition on $\Gamma_{0}$ and depends on the base-flow, $\overline{\mathbf{q}}$. The eigenmode is therefore a function of both $\Gamma_{0}$ and $\overline{\mathbf{q}}$, i.e. $\hat{\mathbf{q}} \equiv \hat{\mathbf{q}}\left(\Gamma_{0}, \overline{\mathbf{q}}\left(\Gamma_{0}\right)\right)$ and $s \equiv s\left(\Gamma_{0}, \overline{\mathbf{q}}\left(\Gamma_{0}\right)\right)$. 
$\Omega$

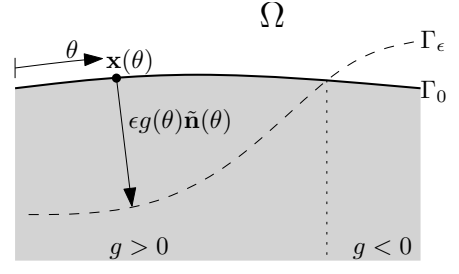

Figure 2: The surface $\Gamma_{0}$ is given by $\mathbf{x}(\theta)$, a function of the surface coordinate, $\theta$. The subsequent deformation onto $\Gamma_{\epsilon}$ is by the small motion $\epsilon g(\theta)$ in the direction of the unit normal, $\tilde{\mathbf{n}}(\theta)$. The unit normal is defined as pointing out of the domain, $\Omega$, so a positive deformation $(g>0)$ deforms the shape inwards, whilst a negative deformation $(g<0)$ deforms the shape outwards.

\subsection{Deformation of $\Gamma_{0}$}

Points lying on the no-slip surface, $\Gamma_{0}$, are given by $\mathbf{x}(\theta)$, a function of the surface coordinate, $\theta$. We create the deformed surface, $\Gamma_{\epsilon}$, by moving each point on $\Gamma_{0}$ a small amount $\epsilon g(\theta)$ in the direction of the surface normal (figure 2 ). Whilst deforming the surface, we keep fixed both the inlet velocity, $\mathbf{u}_{+}$, and the locations of the inlet, outlet and perfect-slip boundaries.

We now consider how the eigenmode changes as we deform $\Gamma_{0}$. A Taylor expansion of the eigenmode gives:

$$
\hat{\mathbf{q}}\left(\Gamma_{\epsilon}, \overline{\mathbf{q}}\left(\Gamma_{\epsilon}\right)\right)=\underbrace{\hat{\mathbf{q}}\left(\Gamma_{0}, \overline{\mathbf{q}}\left(\Gamma_{0}\right)\right)}_{\hat{\mathbf{q}}_{0}}+\epsilon \underbrace{\frac{\partial \hat{\mathbf{q}}}{\partial \Gamma_{0}} \circ g}_{\hat{\mathbf{q}}_{1}^{F}}+\epsilon \underbrace{\frac{\partial \hat{\mathbf{q}}}{\partial \overline{\mathbf{q}}} \circ \overline{\mathbf{q}}_{1}}_{\hat{\mathbf{q}}_{1}^{B}}+O\left(\epsilon^{2}\right) ;
$$

$$
s\left(\Gamma_{\epsilon}, \overline{\mathbf{q}}\left(\Gamma_{\epsilon}\right)\right)=\underbrace{s\left(\Gamma_{0}, \overline{\mathbf{q}}\left(\Gamma_{0}\right)\right)}_{s_{0}}+\epsilon \underbrace{\frac{\partial s}{\partial \Gamma_{0}} \circ g}_{s_{1}^{F}}+\epsilon \underbrace{\frac{\partial s}{\partial \overline{\mathbf{q}}} \circ \overline{\mathbf{q}}_{1}}_{s_{1}^{B}}+O\left(\epsilon^{2}\right) ;
$$

where the first order response of the eigenfunction and eigenvalue has been split into (i) a component due to changing $\Gamma_{0}$ with $\overline{\mathbf{q}}$ fixed: $\hat{\mathbf{q}}_{1}^{F}$ and $s_{1}^{F}$, and (ii) a component due to changing $\overline{\mathbf{q}}$ with $\Gamma_{0}$ fixed: $\hat{\mathbf{q}}_{1}^{B}$ and $s_{1}^{B}$. The component $s_{1}^{F}$ will be referred to as the feedback contribution to the eigenvalue response because it is caused by changes in the unsteady feedback mechanism of the mode. The component $s_{1}^{B}$ will be referred to as the base-flow contribution to the eigenvalue response because it is caused by the change in base-flow caused by deforming $\Gamma_{0}$. The change in base-flow, $\overline{\mathbf{q}}_{1}$, is defined by the Taylor expansion of the base-flow:

$$
\overline{\mathbf{q}}\left(\Gamma_{\epsilon}\right)=\underbrace{\overline{\mathbf{q}}\left(\Gamma_{0}\right)}_{\overline{\mathbf{q}}_{0}}+\epsilon \underbrace{\frac{\partial \overline{\mathbf{q}}}{\partial \Gamma_{0}} \circ g}_{\overline{\mathbf{q}}_{1}}+O\left(\epsilon^{2}\right)
$$

\subsection{Evaluation of the feedback contribution to the eigen- value response, $s_{1}^{F}$}

We substitute the Taylor expansions, (3) and (4), into the governing equations, (1) and (2), and collect terms at order $\epsilon$. The governing equations for the feedback contribution to the eigenmode response are:

$$
\begin{gathered}
s_{0}\left(\begin{array}{c}
\hat{\mathbf{u}}_{1}^{F} \\
0
\end{array}\right)+s_{1}^{F}\left(\begin{array}{c}
\hat{\mathbf{u}}_{0} \\
0
\end{array}\right)+\mathcal{L} \hat{\mathbf{q}}_{1}^{F}=0 ; \\
\hat{\mathbf{u}}_{1}^{F}=0 \text { on } \Gamma_{+} ; \hat{p}_{1}^{F} \tilde{\mathbf{n}}-\nu \frac{\partial \hat{\mathbf{u}}_{1}^{F}}{\partial n}=0 \text { on } \Gamma_{-} ; \\
\frac{\partial \hat{\mathbf{u}}_{1}^{F}}{\partial n} \cdot \tilde{\boldsymbol{\tau}}=\hat{\mathbf{u}}_{1}^{F} \cdot \tilde{\mathbf{n}}=0 \text { on } \Gamma_{s} ; \hat{\mathbf{u}}_{1}^{F}=-g \frac{\partial \hat{\mathbf{u}}_{0}}{\partial n} \text { on } \Gamma_{0} ;
\end{gathered}
$$

where the boundary conditions on the fixed boundaries are the linearized boundary conditions of (2c). The boundary condition on $\Gamma_{0}$ comes from referring the no-slip condition on the deformed surface, $\Gamma_{\epsilon}$, back to the undeformed surface, $\Gamma_{0}$. It states that, at first order, deforming the surface appears as a change in the mode's oscillating velocity at $\Gamma_{0}$. This change in velocity is tangential to the surface and proportional to the local shear-stress.

The feedback contribution to the eigenvalue response is a result of forcing the eigenmode's governing equations by this change in tangential velocity at the no-slip boundary. The forced equations (5) are degenerate, admitting solutions $\hat{\mathbf{q}}_{1}^{F}$ containing an arbitrary amount of $\hat{\mathbf{q}}_{0}$. To determine $s_{1}^{F}$ a compatibility condition must be satisfied. Applying the compatibility condition requires use of the adjoint eigenmode, $\left(\hat{\mathbf{q}}_{0}^{\dagger}, s_{0}^{*}\right)$, which represents the receptivity of the eigenvalue to a forcing of the eigenmode's governing equations. The adjoint eigenmode is defined by:

$$
\begin{gathered}
s_{0}^{*}\left(\begin{array}{c}
\hat{\mathbf{u}}_{0}^{\dagger} \\
0
\end{array}\right)+\mathcal{L}^{\dagger} \hat{\mathbf{q}}_{0}^{\dagger}=0 \\
\left\langle\hat{\mathbf{u}}_{0}^{\dagger}, \hat{\mathbf{u}}_{0}\right\rangle_{\Omega}=1 \\
\hat{\mathbf{u}}_{0}^{\dagger}=0 \text { on } \Gamma_{+} ; \quad \hat{p}_{0}^{\dagger} \tilde{\mathbf{n}}+\nu \frac{\partial \hat{\mathbf{u}}_{0}^{\dagger}}{\partial n}=-\left(\hat{\mathbf{u}}_{0} \cdot \tilde{\mathbf{n}}\right) \hat{\mathbf{u}}_{0}^{\dagger} \text { on } \Gamma_{-} ; \\
\frac{\partial \hat{\mathbf{u}}_{0}^{\dagger}}{\partial n} \cdot \tilde{\boldsymbol{\tau}}=\hat{\mathbf{u}}_{0}^{\dagger} \cdot \tilde{\mathbf{n}}=0 \text { on } \Gamma_{s} ; \quad \hat{\mathbf{u}}_{0}^{\dagger}=0 \text { on } \Gamma_{0} ;
\end{gathered}
$$

where the operator, $\mathcal{L}^{\dagger}$, is the adjoint of the operator $\mathcal{L}$. The adjoint eigenfunction is normalised by $(6 \mathrm{~b})$.

The compatibility condition is applied by taking the inner product of both sides of (5a) with the adjoint eigenfunction, $\hat{\mathbf{q}}_{0}^{\dagger}$. Repeated application of the divergence theorem allows the feedback contribution of the eigenvalue to be evaluated as:

$$
s_{1}^{F}=\int_{\Gamma_{0}} g \nu \frac{\partial \hat{\mathbf{u}}_{0}}{\partial n} \cdot \frac{\partial \hat{\mathbf{u}}_{0}^{\dagger *}}{\partial n} \mathrm{~d} \Gamma
$$

The factor of $\frac{\partial \hat{\mathbf{u}}_{0}^{\dagger *}}{\partial n}$ measures how sensitive the eigenvalue is to a change in the mode's oscillating tangential velocity. The factor of $\frac{\partial \hat{\mathbf{u}}_{0}}{\partial n}$ measures how much the mode's oscillating tangential velocity will change when the surface is deformed. 
2.3. Evaluation of the base-flow contribution to the eigenvalue response, $s_{1}^{B}$

The governing equations for the base-flow contribution to the eigenmode response are:

$$
\begin{gathered}
s_{0}\left(\begin{array}{c}
\hat{\mathbf{u}}_{1}^{B} \\
0
\end{array}\right)+s_{1}^{B}\left(\begin{array}{c}
\hat{\mathbf{u}}_{0} \\
0
\end{array}\right)+\mathcal{L} \hat{\mathbf{q}}_{1}^{B}+\delta \mathcal{L}\left(\overline{\mathbf{q}}_{1}\right) \hat{\mathbf{q}}_{0}=0 ; \\
\hat{\mathbf{u}}_{1}^{B}=0 \text { on } \Gamma_{+} ; \quad \hat{p}_{1}^{B} \tilde{\mathbf{n}}-\nu \frac{\partial \hat{\mathbf{u}}_{1}^{B}}{\partial n}=0 \text { on } \Gamma_{-} ; \\
\frac{\partial \hat{\mathbf{u}}_{1}^{B}}{\partial n} \cdot \tilde{\boldsymbol{\tau}}=\hat{\mathbf{u}}_{1}^{B} \cdot \tilde{\mathbf{n}}=0 \text { on } \Gamma_{s} ; \hat{\mathbf{u}}_{1}^{B}=0 \text { on } \Gamma_{0} .
\end{gathered}
$$

For the base-flow contribution to the eigenvalue response, the effect of deforming $\Gamma_{0}$ appears as a change in base-flow, $\overline{\mathbf{q}}_{1}$. This change in base-flow then forces the eigenmode's governing equations by a change, $\delta \mathcal{L}\left(\overline{\mathbf{q}}_{1}\right)$, to the linear operator, $\mathcal{L}$. The change in linear operator is given by:

$$
\delta \mathcal{L}\left(\overline{\mathbf{q}}_{1}\right) \equiv\left(\begin{array}{cc}
\nabla \star \cdot \overline{\mathbf{u}}_{1}+\nabla \overline{\mathbf{u}}_{1} \cdot \star & 0 \\
0 & 0
\end{array}\right) .
$$

The governing equations for $\hat{\mathbf{q}}_{1}^{B}$ are also degenerate. Compatibility conditions must again be applied using the adjoint eigenmode in order to determine $s_{1}^{B}$, the base-flow contribution to the eigenvalue response. Taking the inner product of both sides with $\hat{\mathbf{q}}_{0}^{\dagger}$ and applying the divergence theorem gives [6]:

$$
\begin{gathered}
s_{1}^{B}=\left\langle\nabla^{B} s, \overline{\mathbf{u}}_{1}\right\rangle_{\Omega} ; \\
\text { where } \nabla^{B} s \equiv-\left(\nabla \hat{\mathbf{u}}_{0}^{*}\right)^{T} \cdot \hat{\mathbf{u}}_{0}^{\dagger}+\nabla \hat{\mathbf{u}}_{0}^{\dagger} \cdot \hat{\mathbf{u}}_{0}^{*} ;
\end{gathered}
$$

where $\nabla^{B} s$ is the sensitivity of the eigenvalue to changes in the base-flow and $\overline{\mathbf{u}}_{1}$ is the change to the base-flow velocity. This sensitivity, $\nabla^{B} s$, is complex valued with its real part, $\nabla^{B} \sigma$, being the sensitivity of the growth-rate to baseflow changes and its imaginary part, $-\nabla^{B} \omega$, being the sensitivity of the frequency to base-flow changes.

\subsubsection{Evaluation of $s_{1}^{B}$ by direct calculation of the change in base-flow, $\overline{\mathbf{q}}_{1}$}

For a given deformation, $g$, the expression (10) can be evaluated after calculating the change in base-flow velocity, $\overline{\mathbf{u}}_{1}$. The governing equations for the change in base-flow, $\overline{\mathbf{q}}_{1}$, are:

$$
\mathcal{L} \overline{\mathbf{q}}_{1}=0 \quad ;
$$

$$
\begin{aligned}
\overline{\mathbf{u}}_{1} & =0 \text { on } \Gamma_{+} ; \quad \bar{p}_{1} \tilde{\mathbf{n}}-\nu \frac{\partial \overline{\mathbf{u}}_{1}}{\partial n}=0 \text { on } \Gamma_{-} ; \\
\frac{\partial \overline{\mathbf{u}}_{1}}{\partial n} \cdot \tilde{\boldsymbol{\tau}} & =\overline{\mathbf{u}}_{1} \cdot \tilde{\mathbf{n}}=0 \text { on } \Gamma_{s} ; \quad \overline{\mathbf{u}}_{1}=-g \frac{\partial \overline{\mathbf{u}}_{0}}{\partial n} \text { on } \Gamma_{0} .
\end{aligned}
$$

To understand better how a change in the base-flow alters the eigenvalue we rewrite (10) as:

$$
\begin{gathered}
\sigma_{1}^{B}=\left\|\nabla^{B} \sigma\right\|_{\Omega}\left\|\overline{\mathbf{u}}_{1}\right\|_{\Omega} \mu_{\sigma}, \quad \omega_{1}^{B}=\left\|\nabla^{B} \omega\right\|_{\Omega}\left\|\overline{\mathbf{u}}_{1}\right\|_{\Omega} \mu_{\omega} ; \\
\text { where } \mu_{\sigma} \equiv\left\langle\tilde{\nabla}^{B} \sigma, \tilde{\mathbf{u}}_{1}\right\rangle_{\Omega}, \quad \mu_{\omega} \equiv\left\langle\tilde{\nabla}^{B} \omega, \tilde{\mathbf{u}}_{1}\right\rangle_{\Omega} ;
\end{gathered}
$$

$$
\text { and }\left|\mu_{\sigma}\right|<1, \quad\left|\mu_{\omega}\right|<1 .
$$

The terms $\tilde{\nabla}^{B} \sigma$ and $\tilde{\nabla}^{B} \omega$ are the normalised growth rate and frequency sensitivities respectively. The term $\tilde{\tilde{\mathbf{u}}}_{1}$ is the normalised change in base-flow velocity. From (12) the changes in growth rate and frequency are a product of: (i) the sensitivity of the growth rate and frequency to base-flow changes, $\left\|\nabla^{B} \sigma\right\|_{\Omega}$ and $\left\|\nabla^{B} \omega\right\|_{\Omega}$; (ii) the size of the change in base-flow velocity, $\left\|\overline{\mathbf{u}}_{1}\right\|_{\Omega}$; and (iii) how effective the base-flow change is at changing the growth rate and frequency, $\mu_{\sigma}$ and $\mu_{\omega}$. In $\S 4$ we examine each of these components in turn in order to explain the structure of the base-flow contribution to the shape sensitivity .

\subsubsection{Evaluation of $s_{1}^{B}$ by calculation of the adjoint base- flow, $\overline{\mathbf{q}}_{0}^{\dagger}$}

Direct calculation of the change in base-flow allows us to understand the physics behind the base-flow response but requires solving (11) for each deformation of interest, $g$. We avoid having to solve (11) for every deformation by introducing an adjoint base-flow, $\overline{\mathbf{q}}_{0}^{\dagger}$, governed by:

$$
\begin{gathered}
\mathcal{L}^{\dagger} \overline{\mathbf{q}}_{0}^{\dagger}+\delta \mathcal{L}^{\dagger}\left(\hat{\mathbf{q}}_{0}\right) \overline{\mathbf{q}}_{0}^{\dagger}=0 \quad ; \\
\overline{\mathbf{u}}_{0}^{\dagger}=0 \text { on } \Gamma_{+} ; p_{0}^{\dagger} \tilde{\mathbf{n}}+\nu \frac{\partial \overline{\mathbf{u}}_{0}^{\dagger}}{\partial n}=\mathbf{A} \text { on } \Gamma_{-} ; \\
\frac{\partial \overline{\mathbf{u}}_{0}^{\dagger}}{\partial n} \cdot \tilde{\boldsymbol{\tau}}=\overline{\mathbf{u}}_{0}^{\dagger} \cdot \tilde{\mathbf{n}}=0 \text { on } \Gamma_{s} ; \overline{\mathbf{u}}_{0}^{\dagger}=0 \text { on } \Gamma_{0} ; \\
\text { where } \mathbf{A}=-\left(\overline{\mathbf{u}}_{0} \cdot \tilde{\mathbf{n}}\right) \overline{\mathbf{u}}_{0}^{\dagger}-\left(\hat{\mathbf{u}}_{0}^{*} \cdot \tilde{\mathbf{n}}\right) \hat{\mathbf{u}}_{0}^{\dagger} .
\end{gathered}
$$

The operator $\delta \mathcal{L}^{\dagger}$ is the adjoint of the operator $\delta \mathcal{L}$.

We add the inner product between the adjoint eigenfunction, $\hat{\mathbf{q}}_{0}^{\dagger}$, and (8) to the inner product between the adjoint base-flow, $\overline{\mathbf{q}}_{0}^{\dagger}$, and (11). After repeated application of the divergence theorem, the governing equations (13) for $\overline{\mathbf{q}}_{0}^{\dagger}$ appear as a result of eliminating terms containing $\overline{\mathbf{q}}_{1}$. Using this definition of $\overline{\mathbf{q}}_{0}^{\dagger}$ allows the base-flow contribution to the eigenvalue response to be evaluated directly from the deformation, $g$, by:

$$
s_{1}^{B}=\int_{\Gamma_{0}} g \nu \frac{\partial \overline{\mathbf{u}}_{0}}{\partial n} \cdot \frac{\partial \overline{\mathbf{u}}_{0}^{\dagger *}}{\partial n} \mathrm{~d} \Gamma
$$

The factor $\frac{\partial \overline{\mathbf{u}}_{0}^{\dagger}}{\partial n}$ measures how sensitive the eigenvalue is to a change in the base-flow's tangential velocity. The factor $\frac{\partial \overline{\mathbf{u}}_{0}}{\partial n}$ measures how much base-flow's tangential velocity will change when the surface is deformed.

\subsection{Overall first order eigenvalue response, $s_{1}$}

We can now add the feedback and base-flow contributions of the eigenvalue response to obtain the overall first order eigenvalue response, $s_{1}=s_{1}^{F}+s_{1}^{B}$ :

$$
s_{1}=\int_{\Gamma_{0}} g(\theta) G(\theta) \mathrm{d} \Gamma \quad,
$$

where $G=G^{F}+G^{B}$;

$$
G^{F} \equiv \nu \frac{\partial \hat{\mathbf{u}}_{0}}{\partial n} \cdot \frac{\partial \hat{\mathbf{u}}_{0}^{\dagger *}}{\partial n} ; \quad G^{B} \equiv \nu \frac{\partial \overline{\mathbf{u}}_{0}}{\partial n} \cdot \frac{\partial \overline{\mathbf{u}}_{0}^{\dagger *}}{\partial n}
$$




\begin{tabular}{|l|c|c|c|c|}
\hline Boundary & Vertex density & $x_{\mathrm{l}}$ & $x_{\mathrm{r}}$ & $y_{\mathrm{c}}$ \\
\hline$\Gamma_{0}$ & 160.0 & - & - & - \\
$B_{0}$ & 20 & -1 & 2 & 1.5 \\
$B_{1}$ & 8 & -5 & 45 & 5 \\
$B_{2}$ & 1.7 & -50 & 100 & 20 \\
$B_{3}$ & 0.25 & -110 & 170 & 60 \\
\hline
\end{tabular}

Table 1: Box coordinates and vertices per unit distance on each surface. The resulting mesh has $\approx 138,000$ vertices and $\approx 278,000$ triangles. The number of vertices on the cylinder surface, $N_{\text {surf }}$, is $N_{\text {surf }}=500$.

The value of $\operatorname{Re}(G)$ at each point, $\mathbf{x}(\theta)$, on $\Gamma_{0}$ represents the sensitivity of the growth rate to a local deformation, $g(\theta)$. If the surface is locally deformed in the direction of its unit normal then the growth rate increases if $\operatorname{Re}(G)$ is positive and decreases if $\operatorname{Re}(G)$ is negative. The $\operatorname{Re}\left(G^{F}\right)$ component is the sensitivity of the growth rate due to the feedback contribution. The $\operatorname{Re}\left(G^{B}\right)$ component is the sensitivity due to the base-flow contribution.

The value of $\operatorname{Im}(G)$ at each $\mathbf{x}(\theta)$ represents the sensitivity of the frequency to local deformations. The $\operatorname{Im}\left(G^{F}\right)$ and $\operatorname{Im}\left(G^{B}\right)$ terms, similarly, are the sensitivities due to the feedback and base-flow contributions to the frequency response.

An inner product for real, scalar functions defined on the surface of $\Gamma_{0}$ is given by:

$$
\langle a, b\rangle_{\Gamma}=\int_{\Gamma_{0}} a b \mathrm{~d} \Gamma
$$

We define the magnitude of a deformation by using the corresponding norm $\|g\|_{\Gamma}=\sqrt{\langle g, g\rangle_{\Gamma}}$. Under this definition of $\|g\|_{\Gamma}, \operatorname{Re}(G)$ becomes the shape gradient of the growth rate; i.e. setting the deformation, $g$, to be a multiple of $\operatorname{Re}(G)$ gives the largest change in growth rate for a given $\|g\|_{\Gamma}$. Similarly, $\operatorname{Im}(G)$ becomes the shape gradient of the frequency. Note that these shape gradients have no constraints on the geometry or any features of the flow other than that the governing equations of the base-flow (1) and the eigenmode (2) must be satisfied.

\section{Application to cylinder flow}

We now consider the flow over a unit cylinder with unit inflow (figure $3 \mathrm{a}$ ) with $R e=50$ based on the cylinder diameter. This is above the critical Reynolds number, $R e_{\mathrm{c}} \approx 47$, for vortex shedding and so we expect the stability analysis to yield a growing oscillating mode $[6,11]$.

The computational domain of figure $3 \mathrm{a}$ is constructed via a Delaunay triangulation using GMSH [20]. The vertex density increases towards the cylinder surface as defined by the series of nested boxes shown in figure $3 \mathrm{~b}$ and table 1. The finite element package FEniCS [21] is used for the spatial discretization using Taylor-Hood elements.
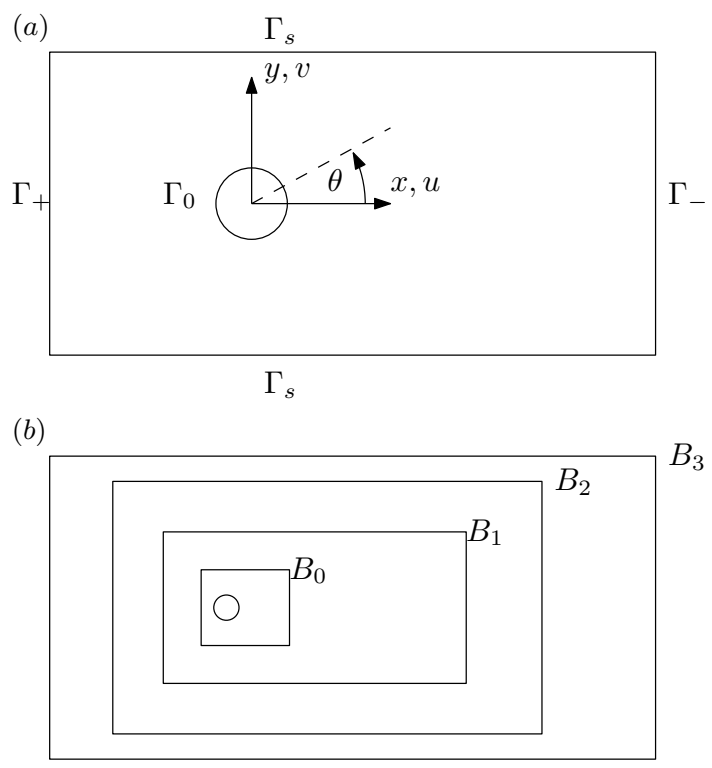

Figure 3: (a) shows the domain, boundaries, coordinate system and definition of the surface coordinate, $\theta \in[-\pi, \pi]$. (b) shows how the computational domain is broken into a series of boxes of increasing vertex density with sides aligned with the streamwise and cross-stream directions. The lower-left and upper-right box corners are located at $\left(x_{1},-y_{\mathrm{c}}\right),\left(x_{\mathrm{r}},+y_{\mathrm{c}}\right)$ and are given in table 1.

The resulting large sparse matrices are manipulated using PETSc [22]. All matrix inversions are performed using the direct LU solver MUMPS [23]. The steady base-flow is found using a Newton-Raphson method. The generalized eigenvalue problem from the direct and adjoint stability problems are solved using SLEPc [24] with a shift-invert method.

The converged base-flow is shown in figure 4a. The global stability analysis reveals that there is only one unstable mode, which has $\sigma=0.0129 \mathrm{~s}^{-1}$ and $\omega=$ $0.737 \mathrm{rad} \mathrm{s}^{-1}$, in good agreement with [6] and [11]. The real and imaginary parts of $\hat{\mathbf{u}}_{0}$ are in spatial quadrature. They extend far downstream from the cylinder as shown
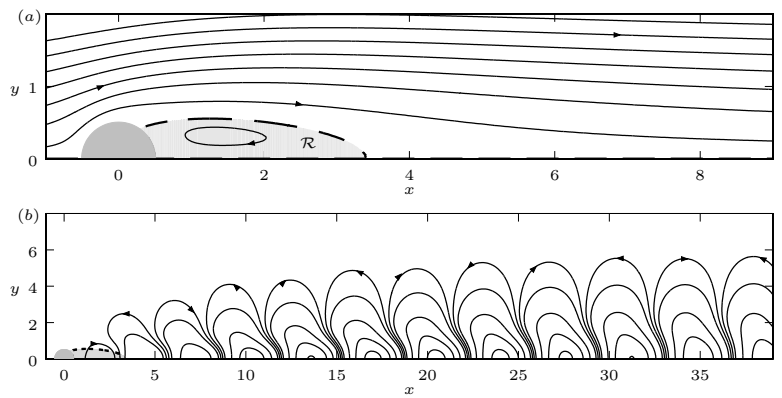

Figure 4: (a) Streamlines of the base-flow plotted as contours of the streamfunction, $\bar{\psi}$. The stagnation streamline, $\bar{\psi}=0$, is dashed. The other streamlines are at $\bar{\psi}=-0.02,0.1,0.3,0.5,0.7,0.9,1.1$ and 1.3. The shaded region is the recirculation bubble, $\mathcal{R}$, defined as the region enclosed by $\bar{\psi}=0$. (b) Streamlines of the most unstable eigenfunction, plotted as contours of the perturbation streamfunction, $\operatorname{Re}(\hat{\psi})$. 


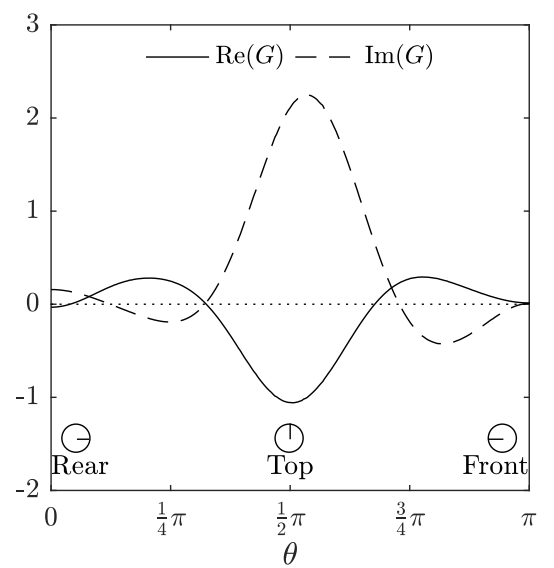

Figure 5: The growth rate sensitivity, $\operatorname{Re}(G)$, and frequency sensitivity, $\operatorname{Im}(\mathrm{G})$, of the most unstable global mode in the flow behind a cylinder. Locally deforming the cylinder inwards increases the growth rate, $\sigma$, where $\operatorname{Re}(G)$ is positive and decreases the growth rate where $\operatorname{Re}(G)$ is negative. Similarly, locally deforming the cylinder inwards increases the frequency, $\omega$, where $\operatorname{Im}(G)$ is positive and decreases the frequency where $\operatorname{Im}(G)$ is negative. The sensitivities are symmetric about the centreline.

in figure $4 \mathrm{~b}$.

\subsection{Shape sensitivity of the eigenvalue}

We apply the analysis of $\S 2$ and calculate the eigenvalue sensitivity, $G$, decomposing it into the growth rate sensitivity, $\operatorname{Re}(G)$, and the frequency sensitivity, $\operatorname{Im}(G)$. The growth rate and frequency are both most sensitive to deformations at the top and bottom of the cylinder and relatively insensitive to deformations at the front and rear of the cylinder (figure 5). This means that deformations which increase or decrease the frontal area of the cylinder lead to a greater eigenvalue response than deformations that keep the frontal area constant. We use two such deformations to validate the sensitivities (figure 6) by comparing the actual eigenvalue response with the predicted eigenvalue response (15).

As well as being the growth rate sensitivity, $\operatorname{Re}(G)$ is also the shape gradient of the growth rate. For a fixed deformation magnitude, $\|g\|_{\Gamma}$, and real constant, $\alpha$, a deformation of the form $g=\alpha \operatorname{Re}(G)$ causes the maximum increase in growth rate if $\alpha>0$ and the maximum decrease in growth growth rate if $\alpha<0$. Similarly, $\operatorname{Im}(G)$, is the shape gradient of the frequency and with fixed $\|g\|_{\Gamma}$ the deformation $g=\alpha \operatorname{Im}(G)$ causes the maximum increase in frequency if $\alpha>0$ and causes the maximum decrease in frequency if $\alpha<0$. Such deformations are shown in figure 7 for $\|g\|_{\Gamma}=0.1$. The deformation that causes the maximum increase in growth rate is a bulge that increases the frontal area of the cylinder. The deformation that causes the maximum increase in frequency is a slightly different bulge in the opposite direction. The shape gradients of the growth rate and frequency have a similar structure but opposite sign i.e. $\frac{\operatorname{Re}(G)}{\|\operatorname{Re}(G)\|_{\Gamma}} \approx-\frac{\operatorname{Im}(G)}{\|\operatorname{Im}(G)\|_{\Gamma}}$. For fixed $\|g\|_{\Gamma}$, this means the deformation that causes the maximum increase

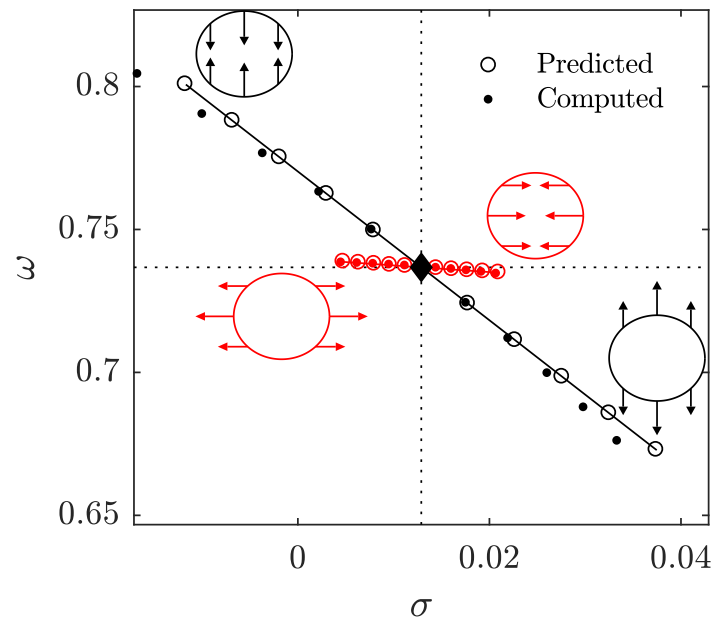

Figure 6: The eigenvalue response as the unit cylinder is deformed into an ellipse by scaling the cylinder in the cross-stream (black) and streamwise (red) directions. The original location of the eigenvalue for the unperturbed cylinder is marked $(\checkmark)$. For deformation magnitudes $\|g\|_{\Gamma}=0.01,0.02,0.03,0.04$ and 0.05 , the position of the new eigenvalue is predicted (o) using the sensitivity analysis of $\S 2$ and computed $(\bullet)$ by repeating the full stability analysis. The loci of the predicted eigenvalue positions are given by the solid lines. This shows that, as expected, the eigenvalue response is predicted accurately by the adjoint methods when $\|g\|_{\Gamma}$ is small. The largest deformation magnitude, $\|g\|_{\Gamma}=0.05$, corresponds to a change in the major-axis of the cylinder of $\approx 10 \%$.

in growth rate is similar to the deformation that causes the maximum decrease in frequency and vice--versa.

\subsection{The feedback, $G^{F}$, and base-flow, $G^{B}$, contributions to the shape sensitivity}

The overall shape sensitivity, $G$, is split into its feedback, $G^{F}$, and base-flow, $G^{B}$, contributions (figure 8). For both the growth rate and the frequency, the feedback contribution is significantly smaller than the base-flow contribution. This means that control of the eigenvalue through deformation of the surface is primarily through changes to the base-flow

The feedback and base-flow contributions are caused by changes in tangential velocity at the no-slip boundary. The feedback contribution is due to the change in the eigenmode's oscillating tangential velocity and the base-flow contribution is due to the change in the base-flow's steady tangential velocity. Unlike eigenvalue control by suction or blowing, where this change in tangential velocity can be arbitrarily chosen by the designer, shape deformation results in changes proportional to the local shear. This gives rise to the factors of the wall-normal gradients of the direct states, $\frac{\partial \hat{\mathbf{u}}_{0}}{\partial n}$ and $\frac{\partial \overline{\mathbf{u}}_{0}}{\partial n}$, appearing in the expressions (15c) for $G^{F}$ and $G^{B}$. The remaining factors in the expressions for $G^{F}$ and $G^{B}$ are the wall-normal gradients of the adjoint states, $\frac{\partial \hat{\mathbf{u}}_{0}^{\dagger}}{\partial n}$ and $\frac{\partial \overline{\mathbf{u}}_{0}^{\dagger}}{\partial n}$, measuring the sensitivity of the eigenvalue to a local change in tangential velocity. The overlap between these wall-normal gradients determines the regions of the surface where the eigenvalue is 


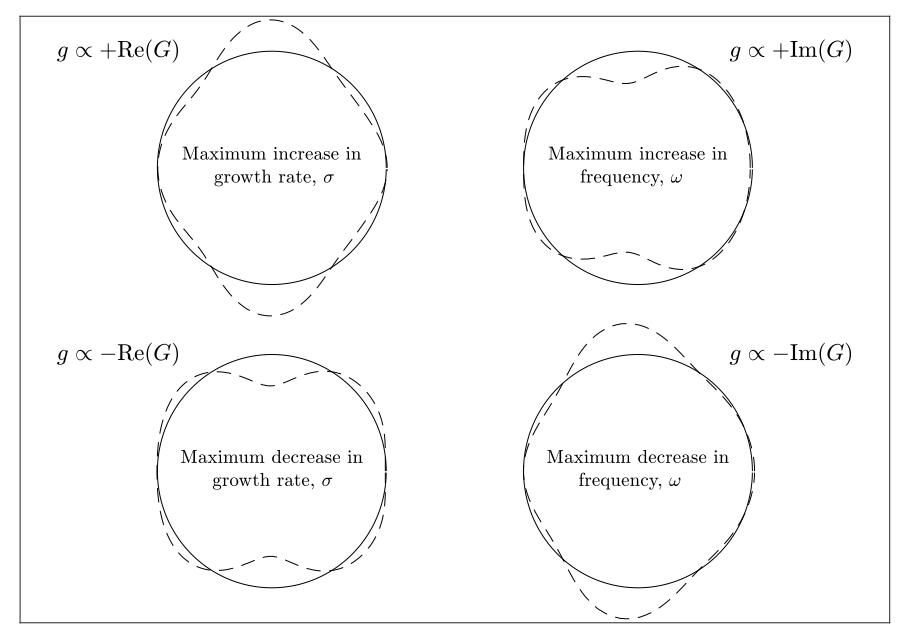

Figure 7: The $\|g\|_{\Gamma}=0.1$ deformations of the unit cylinder that give the maximum increase/decrease in growth rate, $\sigma$, and the maximum increase/decrease in frequency, $\omega$.
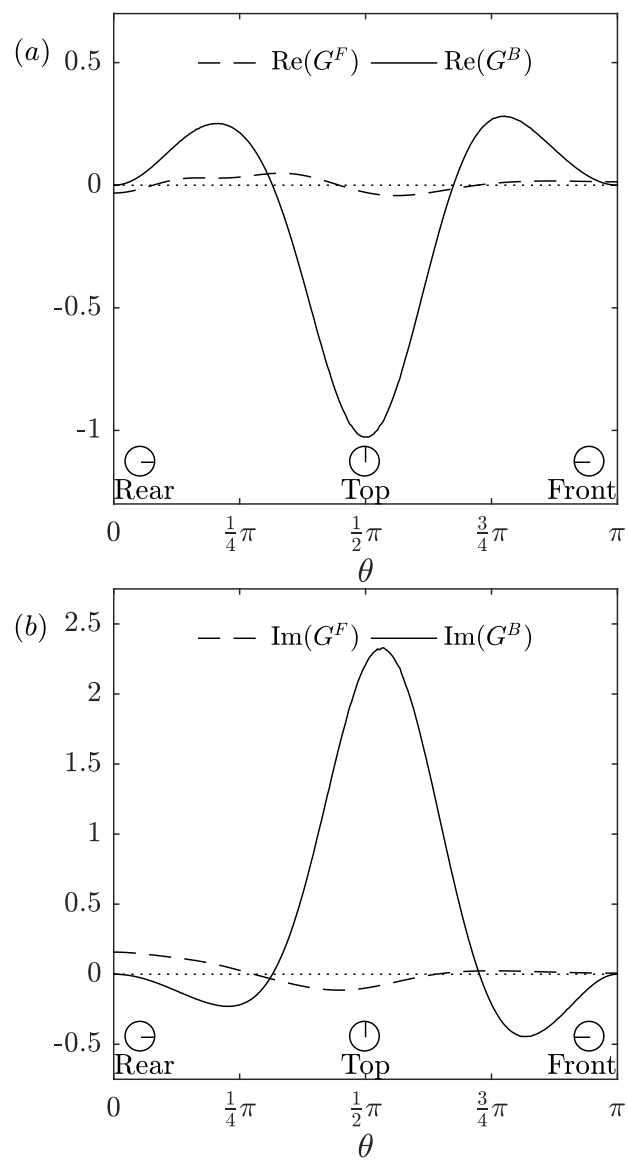

Figure 8: (a) The growth rate sensitivity, $\operatorname{Re}(G)$, and $(b)$ the frequency sensitivity, $\operatorname{Im}(G)$, decomposed into feedback, $G^{F}$, and baseflow, $G^{B}$, contributions. sensitive to surface deformation. The phase difference between these terms determines whether deformations affect the growth rate, $\sigma$, or the frequency, $\omega$.

For the feedback contribution, figure 9 (a i), the wallnormal gradients differ by four orders of magnitude. The eigenfunction has greatest wall-normal gradient (dashed line) at the rear of the cylinder, falling to a minimum near the separation point and remaining low at the front of the cylinder. This makes shape changes at the rear of the cylinder most effective at altering the eigenmode's oscillating tangential velocity. The eigenvalue, however, is most sensitive (dotted line) to changes in the oscillating tangential velocity near the separation point, leading to a sensitivity (solid line) that is largest around the rear of the cylinder.

For the base-flow contribution, figure 9 (a ii), the wallnormal gradients have comparable magnitudes and much greater overlap. Although the eigenvalue is most sensitive to base-flow changes near the separation point where the shear is identically zero, the base-flow shear quickly increases over the front of the cylinder resulting in significant overlap near the top of the cylinder. At the separation point, where the feedback contribution is low and the baseflow contribution is zero, shape deformations provide little control of the eigenvalue. This confirms the observation that the exact geometry around the cylinder's separation point has little influence on the stability of the flow [8].

The phase-difference between the direct and adjoint eigenmode varies continuously across the surface of the cylinder. In contrast, the phase-difference between the direct and adjoint base-flow (figure $9(b)$ ) is approximately piecewise constant. Changes in the phase occur only at the separation point, where the reversal of the base-flow shear causes the phase difference to change discontinuously, and at the front of the cylinder, where the phase of the adjoint base-flow changes rapidly. The remainder of the cylinder has approximately constant phase difference in each region and with a phase difference such that deformations have the opposite effect on the growth rate and frequency. This leads the real part of $G^{B}$ to appear as a scaled and inverted version of the imaginary part. Because the base-flow contribution is the dominant contribution, this is responsible for the shape gradients of the growth rate and frequency having similar structure but opposite sign. This results in similar changes in base-flow (figure 10) when applying a deformation proportional to $\operatorname{Re}\left(G^{B}\right)$, for the greatest increase in growth-rate through base-flow changes, and proportional to $\operatorname{Im}\left(G^{B}\right)$, for the greatest increase in frequency through base-flow changes.

\section{Physical mechanisms behind the baseflow con- tribution to the eigenvalue response, $s_{1}^{B}$}

We now explain the physical mechanisms behind the base-flow contributions to the growth rate and frequency sensitivities and what causes them to have a similar structure. We do this by examining each of the terms giving 

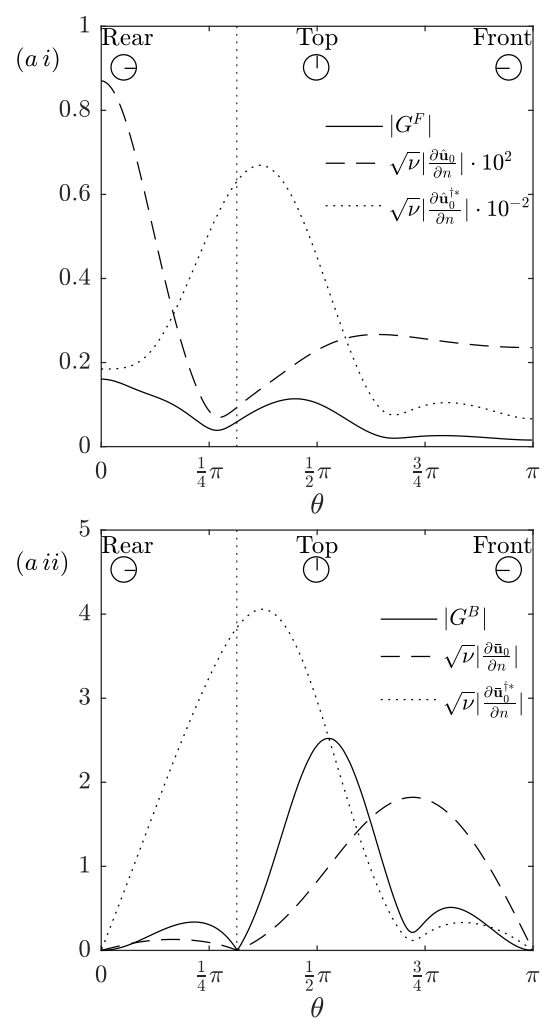

$(b)$

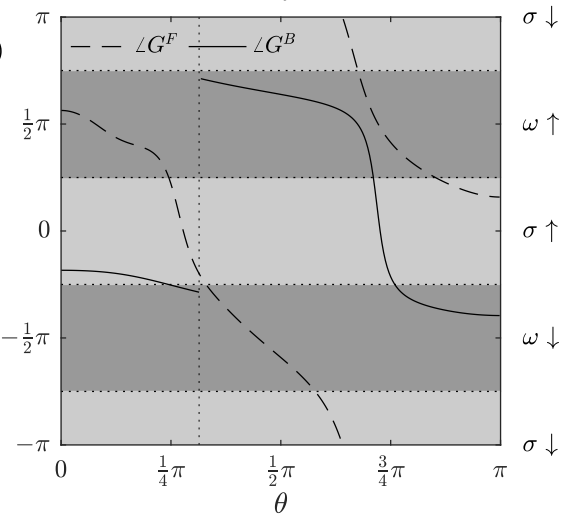

Figure 9: (a) The magnitudes and (b) the arguments of the contributions to the eigenvalue shape sensitivity at each point on the cylinder surface. The location of the separation point is shown by the vertical dashed line. (a $i)$ The feedback contribution, $G^{F}$, split into its factors $\sqrt{\nu} \frac{\partial \hat{\mathbf{u}}_{0}}{\partial n}$ and $\sqrt{\nu} \frac{\partial \hat{\mathbf{u}}_{0}^{\dagger *}}{\partial n}$. Additional factors of $10^{2}$ are included to aid visibility. ( $a$ ii) The base-flow contribution, $G^{B}$, split into its factors $\sqrt{\nu} \frac{\partial \overline{\mathbf{u}}_{0}}{\partial n}$ and $\sqrt{\nu} \frac{\partial \overline{\mathbf{u}}_{0}}{\partial n}$. (b) The complex arguments of $G^{F}$ and $G^{B}$ and whether they mainly act to increase ( $\uparrow$ ) or decrease $(\downarrow)$ the growth-rate, $\sigma$, and frequency, $\omega$.

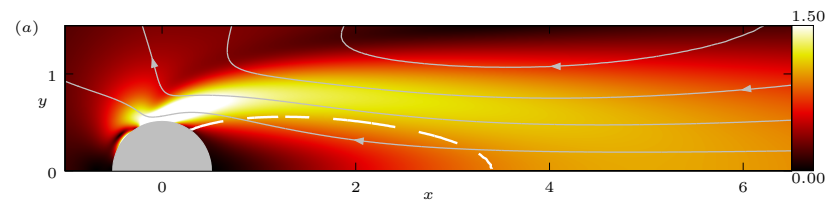

(b)

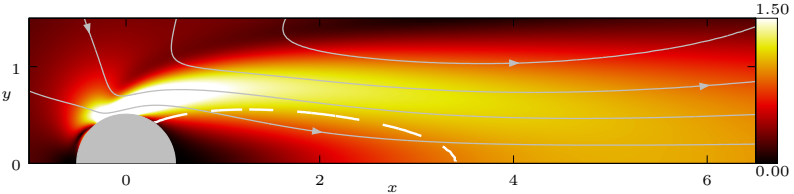

Figure 10: The change in base-flow velocity, $\overline{\mathbf{u}}_{1}$, due to a unit deformation, $g$, in the direction of the base-flow contributions to the shape gradient of $(a)$ the growth-rate, giving $g=\frac{\operatorname{Re}\left(G^{B}\right)}{\left\|\operatorname{Re}\left(G^{B}\right)\right\|_{\Gamma}}$, and (b) the frequency, giving $g=\frac{\operatorname{Im}\left(G^{B}\right)}{\left\|\operatorname{Im}\left(G^{B}\right)\right\|_{\Gamma}}$.

rise to the base-flow contribution: (i) the sensitivity of the growth rate and frequency to base-flow changes, $\left\|\nabla^{B} \sigma\right\|_{\Omega}$ and $\left\|\nabla^{B} \omega\right\|_{\Omega}$; (ii) the size of the change in base-flow velocity, $\left\|\overline{\mathbf{u}}_{1}\right\|_{\Omega}$; and (iii) the effectiveness of the base-flow velocity change at changing the growth rate and frequency, $\mu_{\sigma}$ and $\mu_{\omega}$.

\subsection{Sensitivity of the eigenvalue to base-flow changes, $\nabla^{B} s$}

The sensitivity of the eigenvalue to an arbitrary change in base-flow velocity is given by $\nabla^{B} s$. Because this includes the sensitivity to changes in base-flow velocity that are not divergence-free, $\nabla^{B} s$ is also not divergence-free, containing a component orthogonal to all divergence-free vector fields. We give $\nabla^{B} s$ a more physically meaningful interpretation by replacing it in all the previous definitions with its projection onto the space of divergence-free vector fields.

The projected growth rate sensitivity and frequency sensitivity, figure $11 \mathrm{a}$ and figure $11 \mathrm{~b}$, now represent the optimal divergence-free changes to the base-flow velocity in order to alter the growth rate and frequency. They are optimal in the sense that a change in base-flow velocity which is a scalar multiple of these projected sensitivities will produce the greatest change in growth rate and frequency for a given magnitude, $\left\|\overline{\mathbf{u}}_{1}\right\|_{\Omega}$. The effectiveness quantities, $\mu_{\sigma}$, and $\mu_{\omega}$, now represent how close the change in baseflow velocity is to being a scalar multiple of the optimal changes for the growth rate and frequency.

For the growth rate, the optimal change in base-flow velocity is located primarily within the recirculation bubble, $\mathcal{R}$, defined as the region enclosed by the stagnation streamline (figure 4a). For the frequency, the optimal change in base-flow velocity has large components both inside and outside the recirculation bubble. The changes in base-flow velocity caused by deformations proportional to $G^{B}$ are not well aligned with these optimal changes and so are not particularly effective at changing the eigenvalue. 
In figure 11 we also decompose the projected sensitivities into the sensitivity due to changes in the production of perturbations, $\left(\nabla^{B} s\right)_{P}$, and the sensitivity due to changes in the advection of perturbations, $\left(\nabla^{B} s\right)_{A}[6]$. In $\S 4.4$ we use this decomposition to show that deformations proportional to the growth rate and frequency shape gradients modify the eigenvalue primarily by changing how perturbations are advected.

\subsection{Magnitude of the change in base-flow velocity, $\left\|\overline{\mathbf{u}}_{1}\right\|_{\Omega}$}

The change in growth rate and frequency is proportional to the magnitude of the change in base-flow velocity caused by deforming the cylinder. We find a set of orthonormal deformations ranked by the magnitude of the change in base-flow velocity by rewriting (11) as:

$$
\overline{\mathbf{u}}_{1}=\mathcal{C} g
$$

where $\mathcal{C}$ is a linear operator mapping deformations to the velocity component of the change in base-flow.

A generalized singular value decomposition of the discretized form of $\mathcal{C}$ produces a unique set of orthonormal deformation basis functions, $\tilde{g}_{i}$, and a unique set of orthonormal velocity basis functions, $\tilde{\overline{\mathbf{u}}}_{1, i}$. The deformation basis functions, $\tilde{g}_{i}$, and the velocity basis functions, $\tilde{\overline{\mathbf{u}}}_{i}$, are related by:

$$
\begin{aligned}
\lambda_{i} \tilde{\overline{\mathbf{u}}}_{1, i} & =\mathcal{C} \tilde{g}_{i} ; \\
\left\langle\tilde{g}_{i}, \tilde{g}_{j}\right\rangle_{\Gamma}=\left\langle\tilde{\mathbf{u}}_{1, i}, \tilde{\overline{\mathbf{u}}}_{1, j}\right\rangle_{\Omega} & =\left\{\begin{array}{ll}
0 & \text { for } i \neq j \\
1 & \text { for } i=j
\end{array} ;\right.
\end{aligned}
$$

where the $\lambda_{i}^{2}$ are the singular values of $\mathcal{C}$. Physically, $\lambda_{i}^{2}$ is the amplification of the deformation, being the square of the ratio of the size of the change in base-flow velocity to the size of the deformation causing it. If a deformation basis function has a large $\lambda_{i}^{2}$ then it will cause a large change in base-flow velocity. Similarly, a deformation basis-function with a small $\lambda_{i}^{2}$ will cause a small change in the base-flow velocity.

We perform the singular value decomposition, obtaining a set of deformation basis-functions that are purely symmetric or antisymmetric about the centerline. Due to the symmetry of the base-flow and the eigenvalue's sensitivity to changes in base-flow velocity, $\nabla^{B} s$, only symmetric deformations can alter the eigenvalue. The singular values corresponding to the first 15 most amplified deformation basis functions are shown in figure 12 and the shapes of the deformation basis functions and corresponding velocity basis functions are shown in figure 13 .

For the most amplified deformations, the changes in the velocity field take place primarily outside the recirculation bubble. Because the optimal velocity change for altering the growth rate is concentrated in the recirculation bubble, $\mathcal{R}$, we find an additional basis-function used to represent the deformation giving the largest change in velocity within $\mathcal{R}$. This is done by repeating the singular value decomposition using the modified inner product, $\langle\mathbf{a}, \mathbf{b}\rangle_{\mathcal{R}} \equiv \int_{\mathcal{R}} \mathbf{a} \cdot \mathbf{b} \mathrm{d} \Omega$, and orthogonalising against the first fifteen basis functions found using the original inner product. To perform the orthogonalisation we use a GramSchmidt orthogonalisation process. This recirculation deformation basis function, $\tilde{g}_{\mathcal{R}}$, has a very low amplification (figure 12) meaning it does not cause a large change in base-flow velocity. The recirculation velocity basis function, $\tilde{\overline{\mathbf{u}}}_{1, \mathcal{R}}$, has a significant component located within the recirculation bubble (figure 13 ) and produces what is effectively a 'base-bleed'.

We project the real and imaginary parts of the baseflow contribution to the shape gradient, $G^{B}$, onto the set of basis functions using the surface inner product, $\langle\cdot, \cdot\rangle_{\Gamma}$. This shows how much each basis function contributes to the base-flow contributions to the growth rate, $\operatorname{Re}\left(G^{B}\right)$, and frequency, $\operatorname{Im}\left(G^{B}\right)$. For the frequency, the most amplified basis function, $\tilde{g}_{0}$, is responsible for almost all of the base-flow contribution to the shape gradient. For the growth-rate, although the most amplified basis function is responsible for most of the base-flow contribution, there are still noticeable $(>1 \%)$ contributions from the second most amplified basis function, $\tilde{g}_{1}$, and the recirculation basis function, $\tilde{g}_{\mathcal{R}}$.

\subsection{Effectiveness}

The effectiveness of each of the velocity basis functions at changing the growth rate and frequency is shown in figure 15. The least amplified basis function, $\tilde{\overline{\mathbf{u}}}_{1, \mathcal{R}}$, is the most effective at altering the growth rate. The most amplified basis function, $\tilde{\overline{\mathbf{u}}}_{1,0}$, is the most effective at changing the frequency.

\subsubsection{Effectiveness at changing the growth rate, $\mu_{\sigma}$}

The local contributions to $\mu_{\sigma}$ from perturbation production, $\mu_{\sigma, P}$, and perturbation advection, $\mu_{\sigma, A}$, are shown in figure 16 . The most amplified basis function, $\tilde{g}_{0}$, acts by changing how perturbations are advected, destabilising the system by advecting perturbations back towards the site of production. This produces an overall destabilisation despite a reduced perturbation production due to weakening of the shear layer.

In contrast, the second most amplified basis function, $\tilde{g}_{1}$, acts by changing how perturbations are produced by strengthening the shear layer. This is complemented by a smaller additional destabilisation due to increased advection of perturbations back towards the site of production.

The most effective basis function at altering the growth rate, $\tilde{g}_{\mathcal{R}}$, does so by strongly reducing the production of perturbations. The change in base-flow velocity has a high velocity region close to and parallel to the centreline. This is 'base-bleed', a change in base-flow velocity normally achieved using blowing. This suppresses the region of absolute instability in the cylinder wake $[25,26]$ primarily by reduction of perturbation production but also by helping to advect perturbations downstream. 

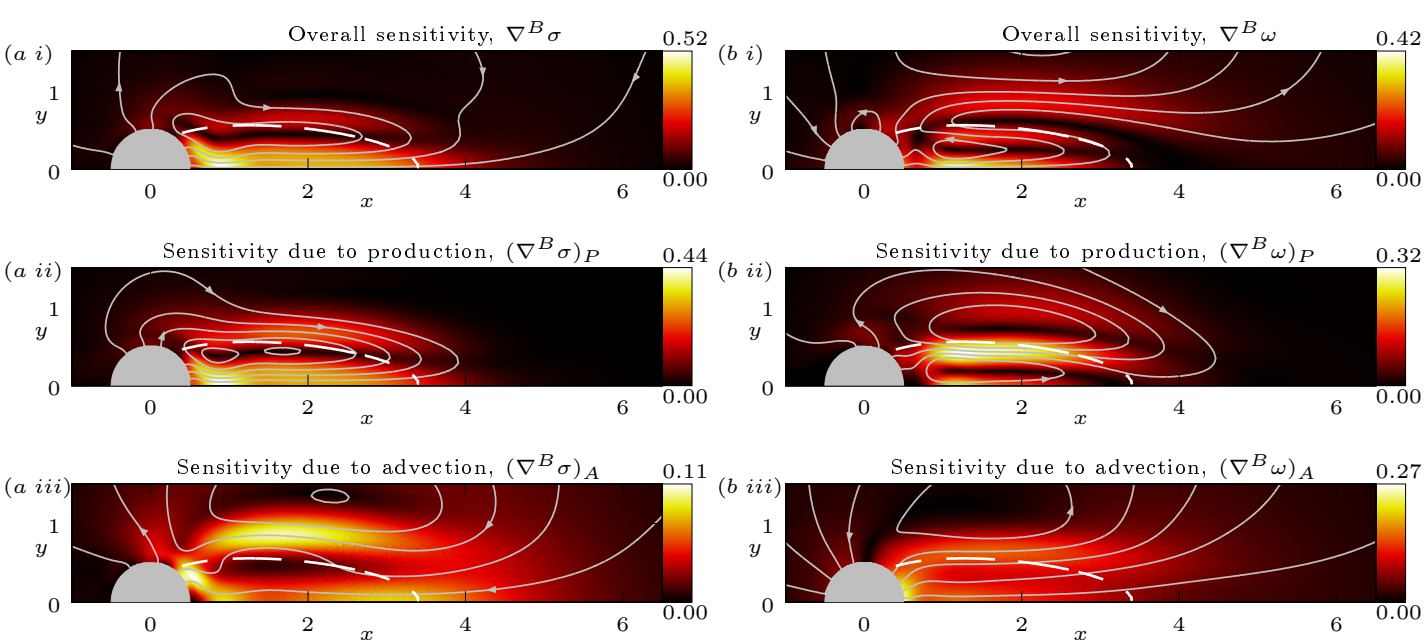

Figure 11: Sensitivity of the growth rate and frequency to changes in base-flow velocity projected onto the space of divergence-free fields. The streamlines show the divergence-free base-flow responses that have the greatest influence on $(a)$ the growth rate and $(b)$ the frequency. The colourscale shows the local influence of the base-flow responses from zero (black) to maximal (white). The overall sensitivity in $(i)$ is split into the components due to $($ ii) production of perturbations and (iii) advection of perturbations 

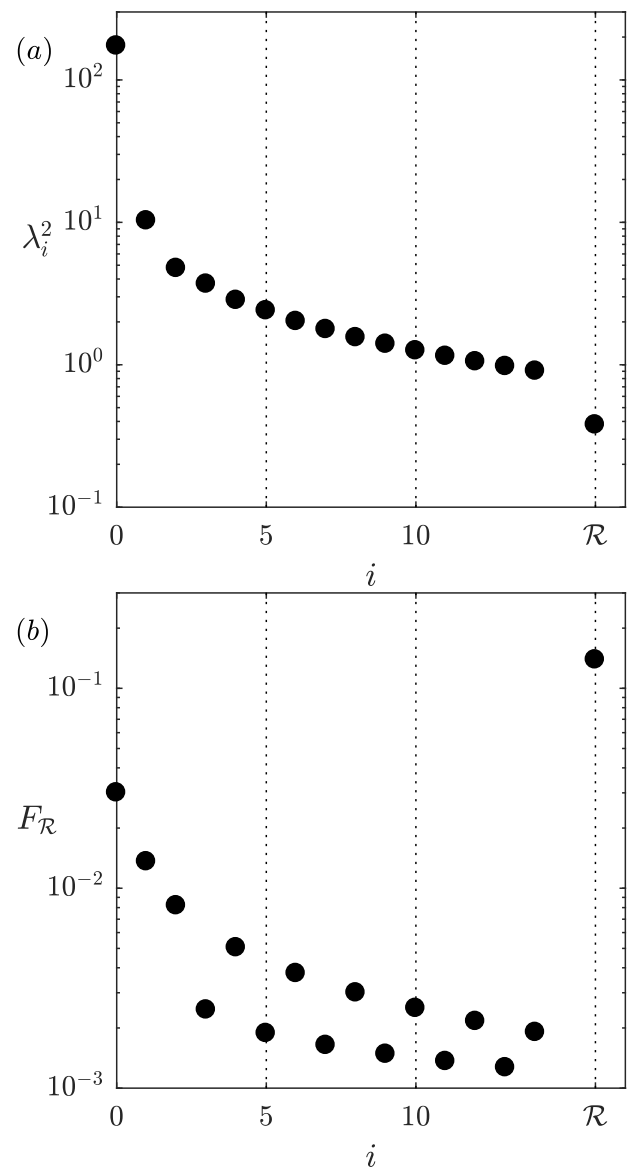

Figure 12: (a) Amplifications, $\lambda_{i}^{2}$, and (b) proportions of the velocity basis-functions within the recirculation bubble, $F_{\mathcal{R}}$, for the first 15 symmetric basis functions, $\tilde{g}_{i}$, (shown by $i=0, \ldots, 14$ ) and for the recirculation basis function, $\tilde{g}_{\mathcal{R}}$, (shown by $i=\mathcal{R}$ ). The amplification is defined by $\lambda_{i}^{2} \equiv \frac{\left\|\overline{\mathbf{u}}_{1, i}\right\|_{\Omega}^{2}}{\left\|\tilde{g}_{i}\right\|_{\Gamma}^{2}}$. The proportion of the velocity basis-function within the recirculation bubble is defined by $F_{\mathcal{R}} \equiv \frac{\int_{\mathcal{R}} \tilde{\mathbf{u}}_{1, i} \cdot \tilde{\mathbf{u}}_{1, i} \mathrm{~d} \Omega}{\int_{\Omega} \tilde{\tilde{\mathbf{u}}}_{1, i} \cdot \tilde{\mathbf{u}}_{1, i} \mathrm{~d} \Omega}$.

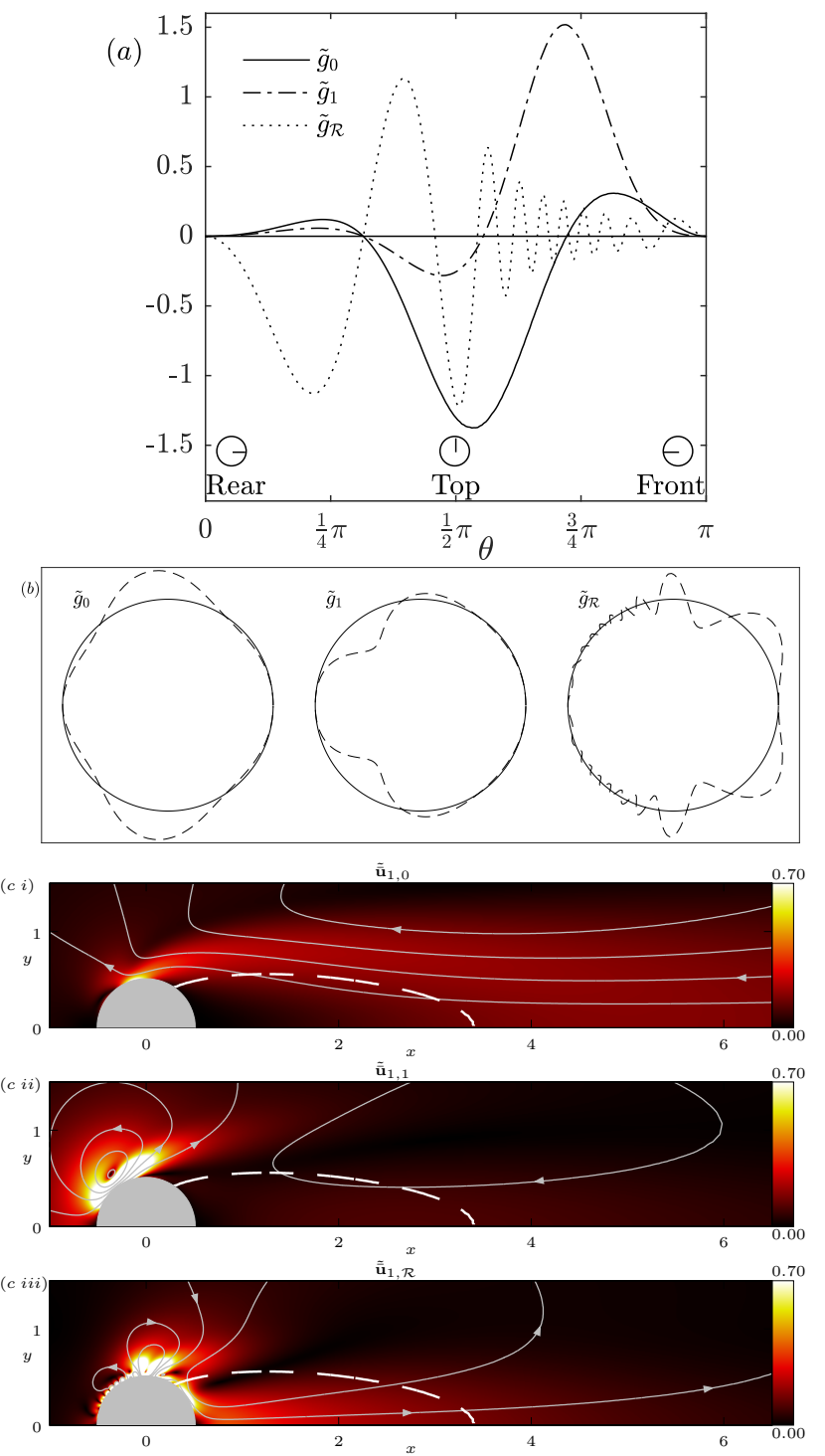

Figure 13: (a) The first 2 deformation basis functions, $\tilde{g}_{i}$, and the recirculation deformation basis function, $\tilde{g}_{\mathcal{R}}$, shown as a function of $\theta$. (b) The morphed unit cylinder when applying deformations of $0.1 \tilde{g}_{i}$. (c) The streamlines of the corresponding velocity basis functions, $\tilde{\mathbf{u}}_{1, i}$. The colour scale shows the local magnitude of $\tilde{\mathbf{u}}_{1, i}$ from zero (black) to maximal (white). 


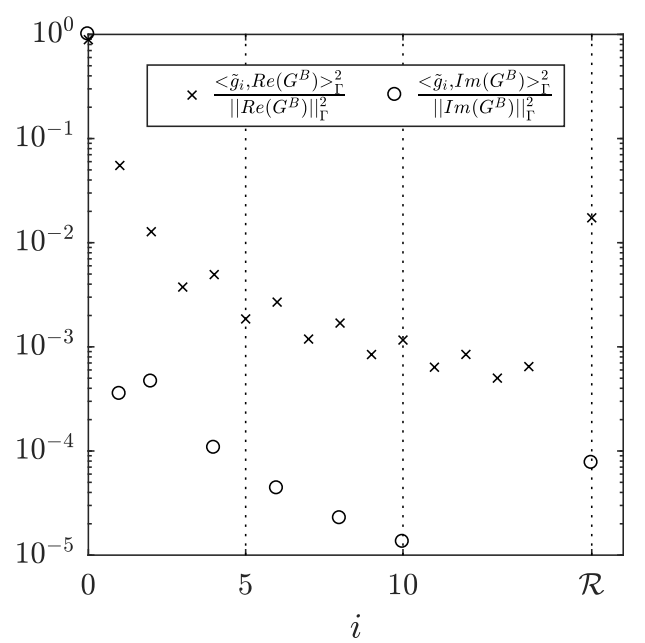

Figure 14: Fraction of the base-flow contribution to the shape gradient of the growth rate, $\operatorname{Re}\left(G^{B}\right)$, and the frequency, $\operatorname{Im}\left(G^{B}\right)$, accounted for by each deformation basis-function, $\tilde{g}_{i}$.

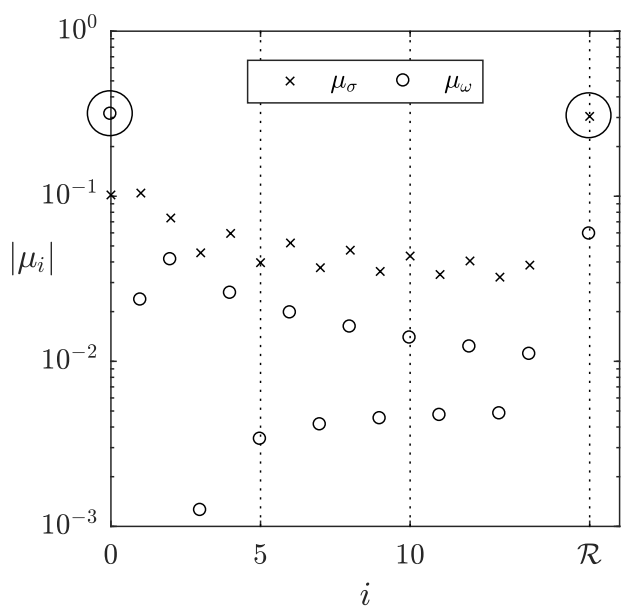

Figure 15: The effectiveness of the basis functions in changing the growth rate, $\left|\mu_{\sigma}\right|$, and the frequency, $\left|\mu_{\omega}\right|$, defined in (12b). The most effective basis functions are circled. 


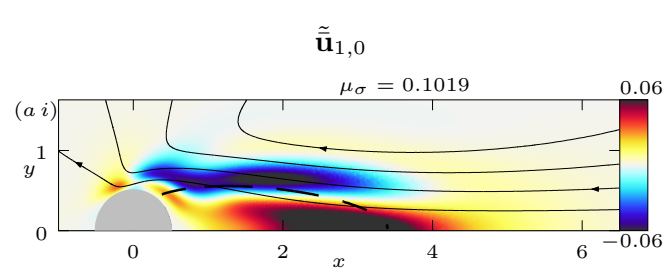

$\tilde{\tilde{\mathbf{u}}}_{1,1}$
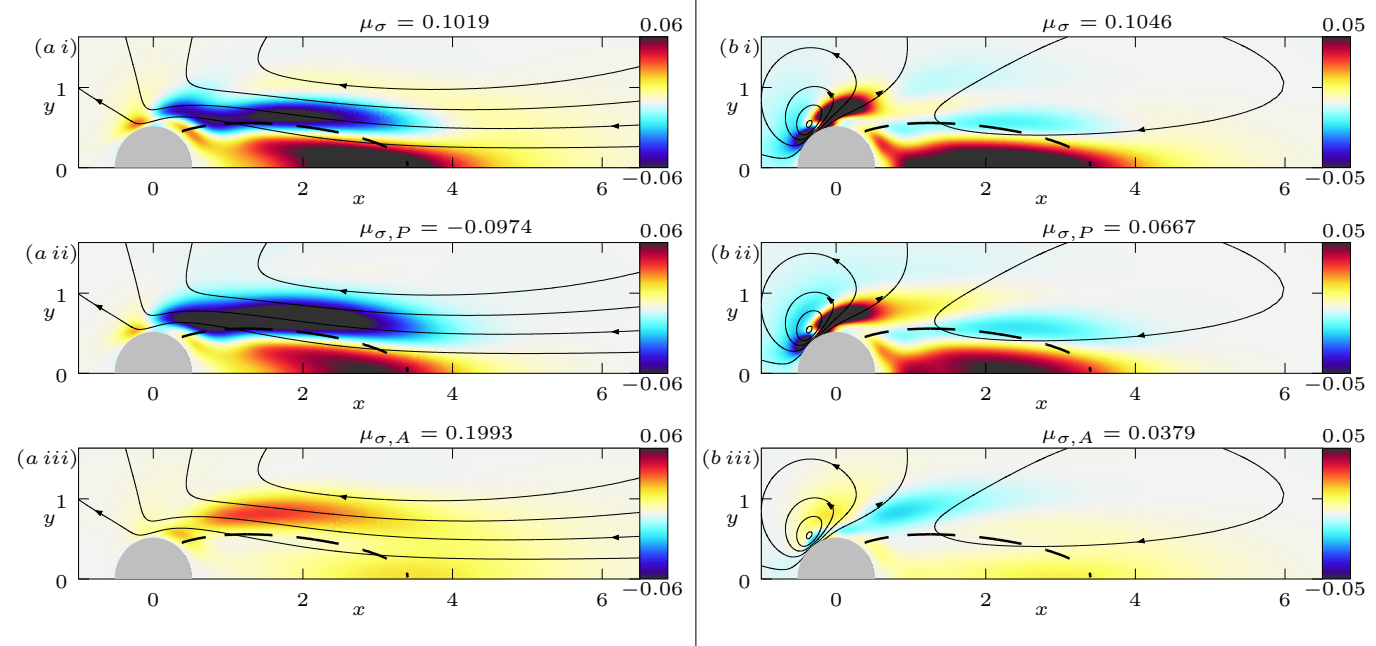
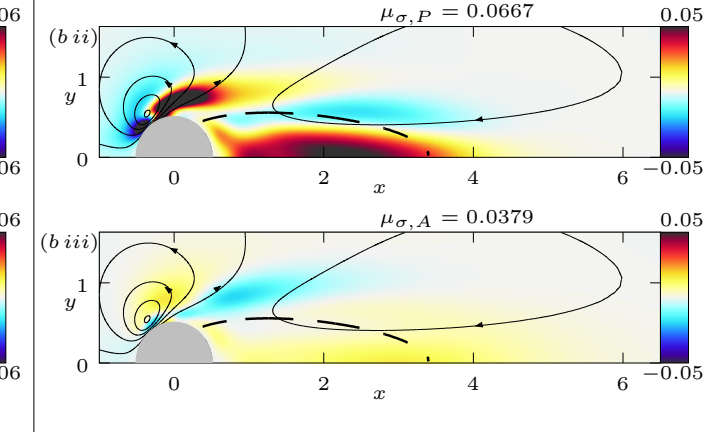

$\tilde{\overline{\mathbf{u}}}_{1, \mathcal{R}}$
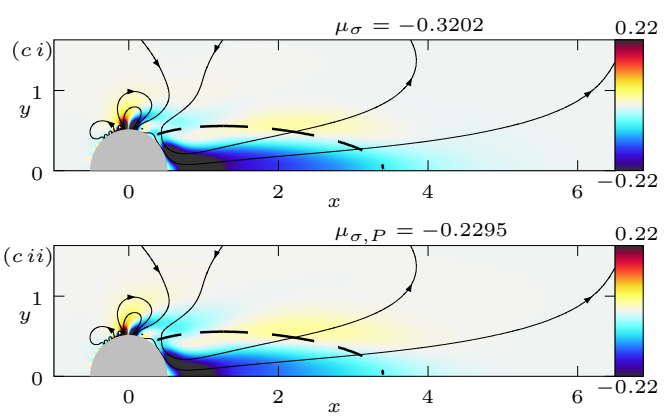

$\mu_{\sigma, A}=-0.0907$

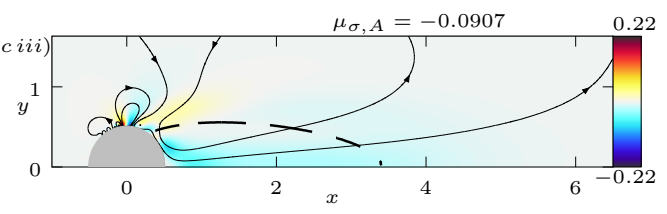

Figure 16: $(a)-(c)$ Streamlines of the velocity basis functions, $\tilde{\mathbf{u}}_{1, i}$. The colour scale shows whether the change in base-flow velocity locally decreases the growth rate (blue) or increases the growth rate (red). The $(i)$ overall change in growth rate is split into contributions from (ii) production of perturbations and (iii) advection of perturbations. The effectiveness, $\mu_{\sigma}$ is similarly split into contributions from the production of perturbations, $\mu_{\sigma, P}$, and advection of perturbations, $\mu_{\sigma, A}$, i.e. $\mu_{\sigma}=\mu_{\sigma, P}+\mu_{\sigma, A}$. The effectiveness is calculated by integrating the colour scale over the whole domain. 

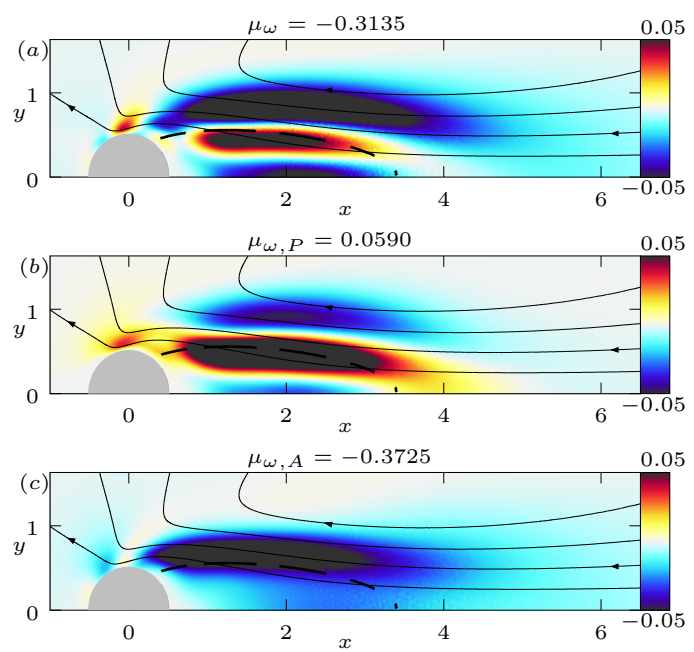

Figure 17: Streamlines of the velocity basis function, $\tilde{\mathbf{u}}_{1,0}$. The colour scale shows whether the change in base-flow velocity locally decreases the frequency (blue) or increases the frequency (red). The (a) overall change in frequency is split into contributions from (b) production of perturbations and $(c)$ advection of perturbations. The effectiveness, $\mu_{\omega}$ is similarly split into contributions from the production of perturbations, $\mu_{\omega, P}$, and advection of perturbations, $\mu_{\omega, A}$, i.e. $\mu_{\sigma}=\mu_{\omega, P}+\mu_{\omega, A}$. The effectiveness is calculated by integrating the colour scale over the whole domain..

\subsubsection{Effectiveness at changing the frequency, $\mu_{\omega}$}

Similar to the previous section we split (figure 17) $\mu_{\omega}$ into a component due to (i) perturbation production, $\mu_{\omega, P}$, and (ii) perturbation advection, $\mu_{\omega, A}$. The most amplified deformation, $\tilde{g}_{0}$, is also the most effective at altering the frequency and so will be the most significant component of the base-flow contribution to the frequency shape gradient. The basis function is highly effective at altering the frequency because it strongly advects the perturbations back towards the cylinder. This results in a large decrease in oscillation frequency, offset slightly from an increase due to modified perturbation production.

\subsection{Overall contribution to the base-flow contribution, $\bar{G}$}

Instead of using the surface inner product, $\langle\cdot, \cdot\rangle_{\Gamma}$, the projection of the base-flow contribution onto the set of deformation basis functions can instead be calculated by combining the individual terms examined in the previous sections:

$$
\operatorname{Re}(\bar{G})=\sum_{i} \beta_{\sigma, i} \tilde{g}_{i}, \quad \operatorname{Im}(\bar{G})=\sum_{i} \beta_{\omega, i} \tilde{g}_{i}
$$

where $\beta_{\sigma, i} \equiv\left\|\nabla^{B} \sigma\right\|_{\Omega} \lambda_{i} \mu_{\sigma, i}, \quad \beta_{\omega, i} \equiv\left\|\nabla^{B} \omega\right\|_{\Omega} \lambda_{i} \mu_{\omega, i}$.

The fraction that each deformation makes up of the baseflow contribution to the shape gradient is given in table 2.

Because, $\tilde{g}_{0}$, is the most amplified deformation basis function by an order of magnitude, it makes up the majority of the base-flow contribution to both the growth rate

\begin{tabular}{|c|c|c|}
\hline Basis & $\frac{\beta_{\sigma, i}^{2}}{\|\operatorname{Re}(\bar{G})\|_{\Omega}^{2}}$ & $\frac{\beta_{\omega, i}^{2}}{\|\operatorname{Im}(\bar{G})\|_{\Omega}^{2}}$ \\
\hline$\tilde{g}_{0}$ & 0.8901 & 0.9986 \\
$\tilde{g}_{1}$ & 0.0552 & 0.0004 \\
$\tilde{g}_{\mathcal{R}}$ & 0.0186 & 0.0001 \\
\hline
\end{tabular}

Table 2: Fractional contribution of each deformation basis function to the base-flow contribution to the sensitivity of the growth rate, $\frac{\beta_{\sigma, i}^{2}}{\|\operatorname{Re}(\bar{G})\|_{\Omega}^{2}}$, and the frequency, $\frac{\beta_{\omega, i}^{2}}{\|\operatorname{Im}(\bar{G})\|_{\Omega}^{2}}$.

shape gradient and to the frequency shape gradient. This leads to the observed similarities between the shape gradients. The mechanism by which $\tilde{g}_{0}$ alters the growth rate and frequency is by advection of perturbations back towards the cylinder. This increases the growth rate but decreases the frequency, leading to the different signs of the growth rate and frequency shape gradients.

Because $\tilde{g}_{0}$ is also the most effective deformation at altering the frequency, it makes up virtually all of the baseflow contribution to the frequency shape gradient. In contrast, for the growth rate, the second most amplified deformation basis function, $\tilde{g}_{1}$, and the recirculation basis function, $\tilde{g}_{\mathcal{R}}$, are both more effective than $\tilde{g}_{0}$. This means that the base-flow contribution to the growth rate shape gradient has noticeable contributions from $\tilde{g}_{1}$ and $\tilde{g}_{\mathcal{R}}$. These additional contributions lead to the subtle differences in the growth rate and frequency shape gradients at the top and at the rear of the cylinder.

\section{Conclusion}

This paper concerns the growth rate and frequency of hydrodynamic oscillations in the wake of a cylinder at $R e=50$. This is a well-known canonical flow, which is often used to discover fundamental behaviour in bluff body flows and to test new numerical techniques. An adjoint method is used to calculate the sensitivity of hydrodynamic oscillations to all possible deformations of the cylinder. This calculation is exact to first order and, crucially, is obtained with just two relatively cheap calculations. It goes significantly beyond previous studies, which only considered the influence of suction and blowing at the cylinder surface or the addition of a second control cylinder.

This paper shows that deformations affect hydrodynamic oscillations mainly through their influence on the steady base-flow, rather than through their influence on the unsteady feedback mechanism. This is consistent with the results of Marquet et al. [6] and Sipp et. al. [12] who considered the sensitivity to the placement of a control cylinder near the surface.

The deformation that most increases the growth rate is a bulge that increases the frontal area of the cylinder $(g \propto+\operatorname{Re}(G)$ in figure $7 \mathrm{a})$. The deformation that most increases the frequency is a subtly different bulge in the opposite direction $(g \propto+\operatorname{Im}(G)$ in figure 7a). The latter deformation (for frequency) acts entirely through the 
deformation that causes the largest modification to the base-flow everywhere ( $\tilde{g}_{0}$ in figure $\left.13 \mathrm{~b}\right)$. The former deformation (for growth rate) acts mainly through the deformation just described, leading to similarities between the two shape gradients. However, it also has noticeable contributions from subtle deformations at the rear of the cylinder $\left(\tilde{g}_{\mathcal{R}}\right.$ in figure $\left.13 \mathrm{~b}\right)$. Although these deformations only slightly change the base-flow in the recirculation zone ( $\mathcal{R}$ in figure 12), these base-flow changes have a disproportionately large influence on the growth rate $(\mathcal{R}$ in figure 16) through a phenomenon similar to base bleed.

The deformations are also decomposed into their influence through the production and the advection of perturbations. On the one hand, this reveals that the deformation that causes the biggest change to the base-flow acts to increase the growth rate by reducing advection while simultaneously decreasing the growth rate by reducing production (figure 16a). These effects counter each other. On the other hand, the deformation that causes the biggest change to the recirculation region acts to decrease the growth rate by increasing advection while simultaneously decreasing the growth rate by reducing production (figure 16c). These effects reinforce each other. This explains physically why geometry changes that modify the recirculation region have a disproportionately large influence on the growth rate.

The adjoint method developed in this paper shows how to alter the frequency and growth rate of hydrodynamic oscillations by making small changes to the geometry of a cylinder. The method is general and versatile. It can be applied to internal or external flows, and it can be combined with a geometric parametrization and optimization algorithm in order to find, for example, the optimal shape to stabilize a flow. Although the user does not have to understand the underlying physical phenomena in order to use this method, the user can nevertheless interrogate the output to interpret the results physically. This improves physical insight into the behaviour that gives rise to a given optimal shape, and therefore can enable intuitive design changes that may be outside the range of the optimization algorithm or existing geometric parametrization.

Now that adjoint methods for shape optimization of eigenvalues in hydrodynamic stability have been developed, the desirable next step is to apply them to industrial problems. Three problems currently being investigated by this group are the reduction of hydrodynamic noise generated by cyclonic separators [2], the alteration of trailing edge stall characteristics in airfoils, and the control of oscillations in injket print heads [28]. This paper enables a significant change in engineering practice in all three areas and has great promise for the design of objects that wish to exploit or prevent oscillations in fluid flows.

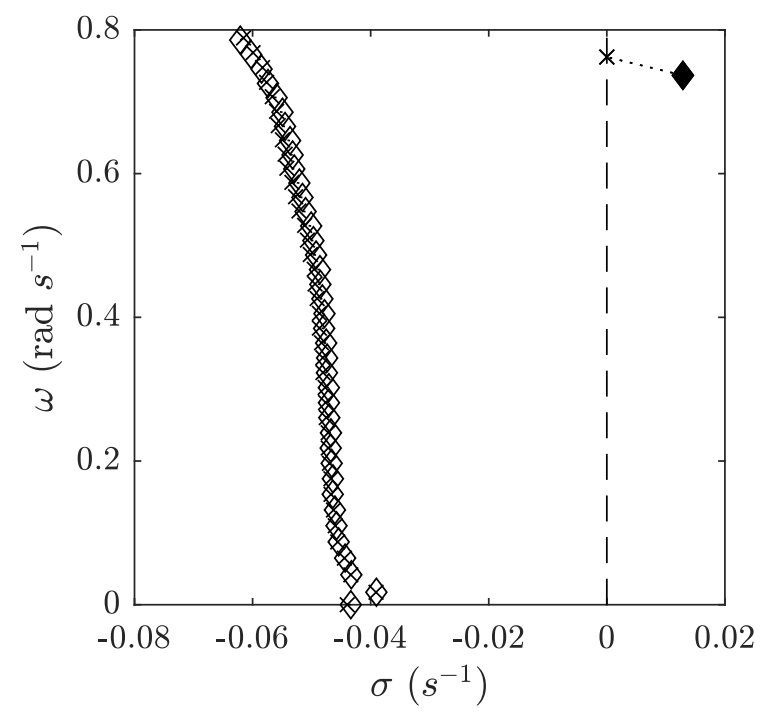

Figure A.18: The response of the eigenvalues to the deformation $g=-\sigma_{0} \frac{\operatorname{Re}(G)}{\|\operatorname{Re}(G)\|_{\Gamma}^{2}}$. This is the linear prediction of the smallest deformation required to make the flow marginally stable. The shifted positions $(x)$ of the unstable global mode $(\downarrow)$ and stable global modes $(\diamond)$ are calculated by applying the linear analysis of $\S 2$ to each mode in turn.

\section{Acknowledgements}

We gratefully acknowledge EPSRC and Dyson Ltd. for funding this project through ICASE studentship 15220080.

\section{Appendix A. Extension to higher Reynolds num- bers}

At higher Reynolds numbers, there may be many unstable modes. If the goal is to create a stable flow, then the shape gradient of each of the unstable modes must be calculated and the deformation that is implemented must stabilise every mode[27]. Furthermore, care must be taken to ensure that modes which were originally stable are not destabilised by the deformations. In figure A.18, this is verified for the case of the cylinder where it is seen that the stable global modes are unaffected by deformations proportional to the unstable mode's shape gradient.

To demonstrate application of this technique at Reynolds numbers further from the critical Reynolds number, $R e_{c} \approx 47$, linear predictions of the smallest deformation required to stabilise the cylinder are presented in figure A.19. The shape of the deformed cylinder is calculated by, at each Reynolds number, performing the stability analysis to get the growth rate, $\sigma(R e)$, and finding the shape gradient of the eigenvalue, $G(R e)$. A deformation proportional to $\operatorname{Re}(G)$ is then used such that $\sigma(R e)+\delta \sigma=0$, where $\delta \sigma$ is the predicted change in $\sigma$ due to application of the shape gradient. 


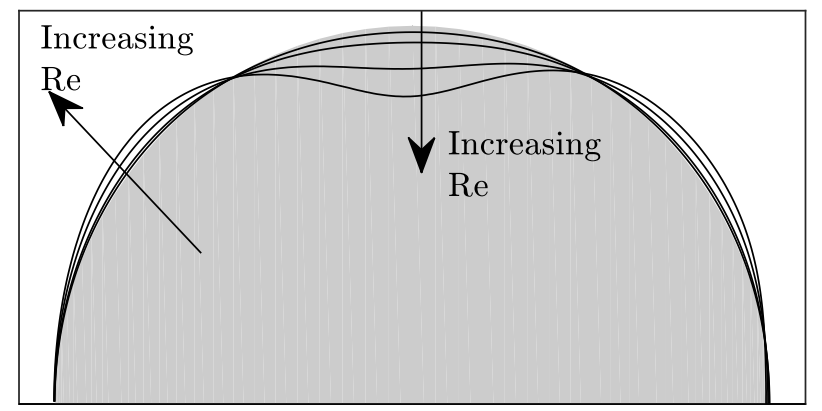

Figure A.19: Cylinders, deformed by direct application of the shape gradient of the growth rate, that are marginally stable at different Re. The unperturbed cylinder (gray) is shown in comparison with the deformed cylinders at $R e=48,50,55$ and 60 . The cylinder is deformed by direct application of the shape gradient. This produces the smallest deformation required to make the flow marginally stable as measured by the surface norm, $\|\cdot\|_{\Gamma}$.

\section{References}

[1] Venugopal, A., Agrawal, A., Prabhu, S. V. 2011 Review on vortex flowmeter-Designer perspective Sensors and Actuators A: Physical 170 (1-2), 8-23

[2] Grimble, T. A., Agarwal, A. \& Juniper, M. P. 2017 Local linear stability analysis of cyclone separators J. Fluid Mech. 816, $507-538$

[3] Heuveline, V. \& Strauss, F. 2009 Shape optimization towards stability in constrained hydrodynamic systems J. Comp. Phys. 228, 938-951

[4] Nakazawa, T. \& Azegami. H 2016 Shape optimization of flow field improving hydrodynamic stability. Japan J. Indust. Appl. Math. 33 (1), 167-181

[5] Kiriyama, Y., Katamine, E. \& Azegami, H. Shape optimisation problem for stability of Navier-Stokes flow field. International Journal of Computational Fluid Dynamics 32 (2-3), 68-87

[6] Marquet O., Sipp, D. \& Jacquin, L. 2008 Sensitivity analysis and passive control of cylinder flow J. Fluid Mech. 615, 221252

[7] Koch, W. 1985 Local instability characteristics and frequency determination of self-excited wake flows J. Sound Vib. 99 (1), 53-83

[8] Huerre, P. \& Monkewitz, P. 1990 Local and global instabilities in spatially developing flows. Annu. Rev. Fluid Mech. 22, 473-537

[9] Huerre, P. 2000 Open shear flow instabilities. In Perspectives in Fluid Mechanics: A Collective Introduction to Current Research (ed. G. K. Batchelor, H. K. Moffat \& M. G. Worster). Cambridge University Press.

[10] HiLl, D. 1992 A theoretical approach for analyzing the restabilization of wakes AIAA Paper 92-0067

[11] Giannetti, F. \& Luchini, P. 2007 Structural sensitivity of the first instability of the cylinder wake J. Fluid Mech. 581, 167-197

[12] Sipp, D., Marquet, O., Meliga, P. \& Barbagallo, A. 2010 Dynamics and control of global instabilities in open-flows: a linearized approach Applied Mechanics Reviews 63 (3)

[13] Tammisola, O., Giannetti, F. , Citro V. \& Juniper, M. P. 2014 Second-order perturbation of global modes and implications for spanwise wavy actuation J. Fluid Mech. 755, 314-335

[14] Meliga, P., Boujo, E. \& Gallaire, F. 2016 A self-consistent formulation for the sensitivity analysis of finite-amplitude vortex shedding in the cylinder wake J. Fluid. Mech. 800, 327-357

[15] JunipeR, M. P. 2012 Absolute and convective instability in gas turbine fuel injectors ASME Turbo Expo, Copenhagen 11-15 June 2012, GT2012-68253
[16] Tammisola, O. \& Juniper, M. P. 2016 Coherent structures in a swirl injector at $R e=4800$ by nonlinear simulations and linear global modes J. Fluid. Mech. 792, 620-657

[17] KaIser, T. 2019 Impact of Flow Rotation on Flame Dynamics and Hydrodynamic Stability Phd Thesis, University of Toulouse, Toulouse

[18] Pironneau, O. 1973 On optimum design in fluid mechanics $J$. Fluid Mech. 64 97-110

[19] Hannemann, K. \& Oertel, H. 1989 Numerical simulation of the absolutely and convectively unstable wake J. Fluid Mech. 199, 55-88

[20] Geuzaine, C. \& Remacle J-F. 2009 Gmsh: A threedimensional finite element mesh generator with built-in preand post-processing facilties Int. J. Numer. Eng. 79 (11), 1309-1331

[21] Alnaes, M. S., Blechta, J., Hake, J., Johansson, A., Kehlet, B., Logg, A., Richardson, C., Ring, J., Rognes, M. E., \& Wells, G. N. 2015 The FEniCS Project Version 1.5 Archive of Numerical Software 3 (100), 9-23

[22] Balay, S., Abhyankar, S., Adams, M., Brown, J., Brune, P., Buschelman, K., Dalcin, L., Eijkhout, V., Gropp, W., Karpeyev, D., Kaushik, D., Knepley, M., May, D., Curfman McInnes, L., Mills, R., Munson, T., Rupp, K., Sanan, P., Smith, B., Zampini, S., Zhang, H. \& Zhang, H. 2018 PETSc Users Manual Argonne National Laboratory

[23] Amestoy, P. R., Duff, I. S., L'Excellent, J-Y., Koster, J. 2001 A fully asynchronous multifrontal solver using distributed dynamic scheduling Siam Journal on Matrix Analysis and Applications 23, 15-41

[24] Hernandez. V., Roman, J. E. \& Vidal, V. 2005 SLEPc: A scalable and flexible toolkit for the solution of eigenvalue problems ACM Trans. Math. Software 31 (3), 351-362

[25] YU, M. \& Monkewitz, P. 1990 The effect of nonuniform density on the absolute instability of two-dimensional inertial jets and wakes. Phys. Fluids A 2, 1175

[26] Hwang, Y. \& Choi, H. 2006 Control of absolute instability by basic-flow modification in a parallel wake at low Reynolds number J. Fluid Mech. 560, 465-475

[27] Agullar, J. G. 2019 Sensitivity analysis and optimization in low order thermoacoustic models PhD Thesis, University of Cambridge

[28] Kungurtsev, P. \& Juniper, M. P. 2019 Adjoint based shape optimization of the microchannels in an inkjet printhead $J$. Fluid Mech. 871, 113-138 